\title{
Thermal correlation functions in CFT and factorization
}

\author{
D. Rodriguez-Gomez ${ }^{a, b}$ and J.G. Russo ${ }^{c, d}$ \\ ${ }^{a}$ Department of Physics, Universidad de Oviedo, \\ C/ Federico García Lorca 18, 33007 Oviedo, Spain \\ ${ }^{b}$ Instituto Universitario de Ciencias y Tecnologías Espaciales de Asturias (ICTEA), \\ C/ de la Independencia 13, 33004 Oviedo, Spain \\ ${ }^{c}$ Institució Catalana de Recerca i Estudis Avançats (ICREA), \\ Pg. Lluis Companys, 23, 08010 Barcelona, Spain \\ ${ }^{d}$ Departament de Física Cuántica i Astrofísica and Institut de Ciències del Cosmos, \\ Universitat de Barcelona, \\ Martí Franquès, 1, 08028 Barcelona, Spain \\ E-mail: d.rodriguez.gomez@uniovi.es, jorge.russo@icrea.cat
}

ABSTRACT: We study 2-point and 3-point functions in CFT at finite temperature for large dimension operators using holography. The 2-point function leads to a universal formula for the holographic free energy in $d$ dimensions in terms of the $c$-anomaly coefficient. By including $\alpha^{\prime}$ corrections to the black brane background, we reproduce the leading correction at strong coupling. In turn, 3-point functions have a very intricate structure, exhibiting a number of interesting properties. In simple cases, we find an analytic formula. When the dimensions satisfy $\Delta_{i}=\Delta_{j}+\Delta_{k}$, the thermal 3-point function satisfies a factorization property. We argue that in $d>2$ factorization is a reflection of the semiclassical regime.

Keywords: AdS-CFT Correspondence, Conformal Field Theory

ARXIV EPRINT: 2105.13909 


\section{Contents}

1 Introduction 1

2 Holographic 3-point functions: setting up the stage 3

3 Thermal 2-point functions in $d$ dimensions $\quad 6$

$\begin{array}{lll}3.1 & \text { Universal formula for the free energy } & 7\end{array}$

3.2 Including $\alpha^{\prime}$ corrections in the $d=4$ case 9

4 Thermal 3-point correlation functions with reflection symmetry $\quad 10$

$\begin{array}{lll}4.1 & \text { Symmetric three-point function in } d=2 & 11\end{array}$

$\begin{array}{ll}4.2 \text { Symmetric three-point function in } d=4 & 12\end{array}$

5 General three-point correlation function and factorization limit $\quad \mathbf{1 3}$

$\begin{array}{lll}5.1 \text { Factorization } & 13\end{array}$

$\begin{array}{lll}5.2 & \text { Adding } \vec{x} \text { dependence } & 15\end{array}$

$\begin{array}{lll}5.3 & \text { Factorization property as a sign of a semiclassical limit } & 17\end{array}$

6 Discussion 18

$\begin{array}{lll}\text { 6.1 Factorization property as a requirement for holography } & 19\end{array}$

$\begin{array}{lll}6.2 & \text { Bootstrap conditions from factorization } & 20\end{array}$

A Details on the geodesic arcs $\quad 20$

$\begin{array}{lll}\text { A.1 The } z_{I} \text { saddle-point equation } & 22\end{array}$

$\begin{array}{lll}\text { A.2 The saddle-point equations for } x_{I} \text { and } \tau_{I} & 23\end{array}$

B Conditions on OPE coefficients from factorization 23

\section{Introduction}

Conformal Field Theories play a pivotal role in Quantum Field Theory as endpoints of trajectories of the Renormalization Group. They also govern the near-critical behavior of phase transitions occurring in relevant models of statistical mechanics and in physical theories. Introducing finite temperature is equivalent to considering a $d$-dimensional CFT placed in euclidean $S^{1} \times \mathbb{R}^{d-1}$, which implies a periodic identification of the euclidean time, $\tau=\tau+\beta$. The response to this non-trivial geometry provides an extra probe of the structure of the theory beyond the usual quantization on $\mathbb{R}^{d}$ (or its conformal cousins such as $\mathbb{R} \times S^{d-1}$ ), which reveals important aspects of the dynamics of the theory.

If one considers two operators inserted within a ball of radius much smaller than $\beta$ - so that effectively they see flat space around - it is possible to use the zero-temperature OPE. 
This was studied in general in [1] (see also [2]) for the case of the 2-point function, finding an elegant expansion in terms of Gegenbauer polynomials, whose coefficients incorporate both the OPE data as well as the non-zero vacuum expectation values in the thermal background. For strongly coupled theories with a gravity dual this structure naturally arises from the computation of the 2-point function in the black brane background, as recently shown for operators of large dimension in [3]. ${ }^{1}$ It is interesting to note that, through the Eigenstate Thermalization Hypothesis, the thermal 2-point function is related to Heavy-Light-Light-Heavy 4-point correlators at $T=0$ (e.g. [5-8]).

The main object of interest in this paper will be thermal (connected) 3-point functions in holographic theories. While at $T=0$ the structure of three-point functions is completely fixed by conformal invariance (just like for 2-point functions), at finite temperature they have an extremely complicated structure, involving multiple regimes.

Holographic $n$-point functions can be computed by means of an expansion in Witten diagrams in the appropriate gravitational background. 3-point functions are distinguished from higher-point functions because there is only one possible Witten diagram, involving a cubic coupling. As a result, the value of the bulk cubic coupling enters only as an overall constant in the final result. Just as in [3], we will focus on correlators of operators with large dimension. In this case, the propagator can be computed in the WKB approximation, where it is represented as the exponential of the geodesic length of the trajectory for a particle moving between the boundary point where the operator is inserted up to the point of interaction. At $T=0$, the 3-point function has been computed using this technique in e.g. [9-11]. A number of new interesting issues arise when extending the method to finite temperature, as we shall discuss.

Thermal correlation functions contain very interesting information about quantum systems including their chaotic nature (see e.g. [12] for a recent application). In holographic theories, it has been shown that they can probe the interior of the black hole in the gravitational dual (e.g. [3, 13-21]). In particular, they are expected to shed new light on the nature of spacetime singularities, although this may require going far beyond the leading strong coupling order. We leave these studies for future work.

This paper is organized as follows. In section 2 we discuss the general set-up for the computation of 3-point functions for operators of large dimension using the geodesic approximation. We define ingoing and returning geodesics and describe the different contributions to the saddle-point equations. Details of the derivations are relegated to appendix A. In section 3 we adapt the discussion to the case of (connected) 2-point functions in $d$-dimensions. By matching with the expected form for the 2-point function in QFT we obtain a formula for the free energy of holographic theories as a function of their central charge $c_{\mathcal{T}}$. Using the known value of the free energy for holographic theories in [22], we test this formula by matching the central charges in $d=3,4,6$ against the known values read off from e.g. [23-25]. For the $d=4$ case, we discuss the leading $\alpha^{\prime}$ correction to the free energy, reproducing the results of [22]. In section 4 we initiate the study of 3-point

\footnotetext{
${ }^{1}$ See also [4] for a similar computation in thermal AdS. This corresponds to a boundary theory with no energy-momentum tensor.
} 
functions, concentrating on a symmetric configuration, for which analytic results can be found. In section 5 we study the general three-point correlation function of scalar operators. We show that in an extremal limit they satisfy a remarkable factorization property. We close in section 6 with a summary and a discussion of implications of the factorization property. Some details of the different calculations are given in appendices.

\section{Holographic 3-point functions: setting up the stage}

We are interested on thermal correlation functions of scalar operators in holographic $d$ dimensional CFT's. In particular, we will examine two-point functions and the three-point function,

$$
\left\langle O_{\Delta_{1}}\left(x_{1}\right) O_{\Delta_{2}}\left(x_{2}\right) O_{\Delta_{3}}\left(x_{3}\right)\right\rangle .
$$

To simplify the problem, we will mostly focus on the case where operators are inserted at the same spatial point, which, with no loss of generality can be taken to be $\overrightarrow{0}$, that is, we will consider $x_{i}=\left(t_{i}, \overrightarrow{0}\right)$.

On general grounds, the finite temperature state is dual to a black brane in $A d S_{d+1}$

$$
d s^{2}=\frac{R^{2}}{z^{2}}\left[-f(z) d t^{2}+\frac{d z^{2}}{f(z)}+d \vec{x}^{2}\right], \quad f(z)=1-\frac{z^{d}}{z_{0}^{d}}, \quad z_{0}=\frac{d}{4 \pi} \beta .
$$

We reserve $t$ for the time coordinate with Lorentzian signature and $\tau$ for the Wick-rotated euclidean coordinate. Each operator is dual to a fluctuating scalar field $\phi_{i}$ in the black brane in $A d S_{d+1}$ with its mass related by the standard holographic formula to the dimension of the dual operator,

$$
\Delta=\frac{d}{2}+\sqrt{\frac{d^{2}}{4}+m^{2} R^{2}} .
$$

We shall consider operators of large scaling dimensions. For such operators, $\Delta \approx m R$ when $m R \gg 1$.

The bulk lagrangian is then obtained by expanding the corresponding gravitational action in the fluctuations, where terms higher-than-quadratic in the fluctuations - suppressed by $\frac{1}{N}$ in terms of CFT variables - give rise to bulk Witten diagrams contributing to higher-point correlators in the boundary theory. Note that 3-point functions are somewhat special in that only cubic vertices contribute to them. Hence the relevant part of the bulk lagrangian looks like

$$
S_{\mathrm{bulk}}=\int d^{d} x \sqrt{g}\left(\frac{1}{2} \partial \phi_{i} \partial \phi_{i}+\frac{1}{2} m_{i}^{2} \phi_{i}^{2}+\lambda_{i j k} \phi_{i} \phi_{j} \phi_{j}\right) .
$$

The $\lambda_{i j k}$ couplings depend on the particular model. However, since such couplings will only appear as overall constants in front of the 3-point function, their precise value will not be relevant in this paper.

In order to compute the 3-point function holographically starting with (2.3) we should evaluate the corresponding 3-leg Witten diagram. Essentially

$$
\left\langle O_{\Delta_{1}}\left(x_{1}\right) O_{\Delta_{2}}\left(x_{2}\right) O_{\Delta_{3}}\left(x_{3}\right)\right\rangle=\lambda_{123} \int d u G_{\Delta_{1}}\left(x_{1}, u\right) G_{\Delta_{2}}\left(x_{2}, u\right) G_{\Delta_{3}}\left(x_{3}, u\right),
$$


where $G_{\Delta_{i}}\left(x_{i}, u\right)$ is the boundary-to-bulk propagator for a field dual to an operator of dimension $\Delta$ from $x_{i}$ in the boundary to the bulk point $u=(\vec{x}, z)$. This propagator comes from solving, with the appropriate boundary conditions, the Klein-Gordon equation in the black brane background. Such computation dramatically simplifies in the case of $\Delta_{i} \gg 1$. In that case one can approximate $G_{\Delta}(x, u)$ by the WKB approximation, which leads to

$$
G_{\Delta}(x, u)=e^{-i S_{\Delta}(x, u)}=e^{-i \Delta \ell(x, u)},
$$

being $S_{\Delta}(x, u)$ the action for a particle of mass $m \sim R^{-1} \Delta$ which departs from the boundary at $x$ and arrives to the bulk point $u$. Alternatively, this can be thought of as the length $\ell(x, u)$ of a geodesic from $x$ to $u$. Thus, for $\Delta_{i} \gg 1$ (but still, $\Delta_{i} \ll c_{\mathcal{T}}$ ), the three-point correlation function of interest can be computed as

$$
\left\langle O_{\Delta_{1}}\left(x_{1}\right) O_{\Delta_{2}}\left(x_{2}\right) O_{\Delta_{3}}\left(x_{3}\right)\right\rangle=\lambda_{123} \int d u e^{-i \Delta_{1} \ell\left(x_{1}, u\right)} e^{-i \Delta_{2} \ell\left(x_{2}, u\right)} e^{-i \Delta_{2} \ell\left(x_{2}, u\right)},
$$

where $u$ now represents the intersecting point of the three geodesic arcs. Under the assumption $\Delta_{i} \gg 1$, the integral over the bulk point $u$ is determined by a saddle-point calculation. Thus, one finds

$$
\left\langle O_{\Delta_{1}}\left(x_{1}\right) O_{\Delta_{2}}\left(x_{2}\right) O_{\Delta_{3}}\left(x_{3}\right)\right\rangle \sim e^{-i S_{\mathrm{os}}},
$$

where $S_{\text {os }}$ stands for the "action" $S=\Delta_{1} \ell\left(x_{1}, u_{I}\right)+\Delta_{2} \ell\left(x_{2}, u_{I}\right)+\Delta_{3} \ell\left(x_{3}, u_{I}\right)$ evaluated on-shell - i.e. at the saddle-point $u_{I}$, solving the equation $d S / d u=0$.

Our first task is to compute the boundary-to-bulk propagator in the black brane background, a.k.a. the geodesic arcs for the case of $\Delta \gg 1$. Since we consider all operators inserted at $\overrightarrow{0}$, it is clear that the spatial part of $u_{I}$ at the saddle point will be at the origin. We can thus focus on geodesics which move in the plane $(z, t)$. The action for a massive particle moving along $t$ whose worldline is parametrized by $z$ is given by

$$
S=-\Delta \int d z \frac{1}{z} \sqrt{f \dot{t}^{2}-\frac{1}{f}},
$$

where $\dot{t}=d t / d z$. Since the action does not depend on $t$, the corresponding conjugate momentum — which will be denoted as $p_{t}=\Delta \mu$ - is conserved. This gives a first-order equation

$$
\dot{\tau}=i \dot{t}= \pm \frac{z \mu}{f \sqrt{f-\mu^{2} z^{2}}},
$$

where we have introduced the euclidean time $\tau=i t$. The signs \pm in (2.9) respectively describe either a geodesic going towards the black hole or a geodesic going towards the boundary.

The parameter $\mu$ can be viewed as an integration constant, which is determined by demanding that the geodesic departs from $\left(\tau=\tau_{1}, z=0\right)$ and reaches the intersection point $\left(\tau_{I}, z_{I}\right)$. At this point,

$$
\tau_{I}=\tau_{1}+\int_{0}^{z_{I}} d z \frac{z \mu}{f(z) \sqrt{f(z)-\mu^{2} z^{2}}}
$$




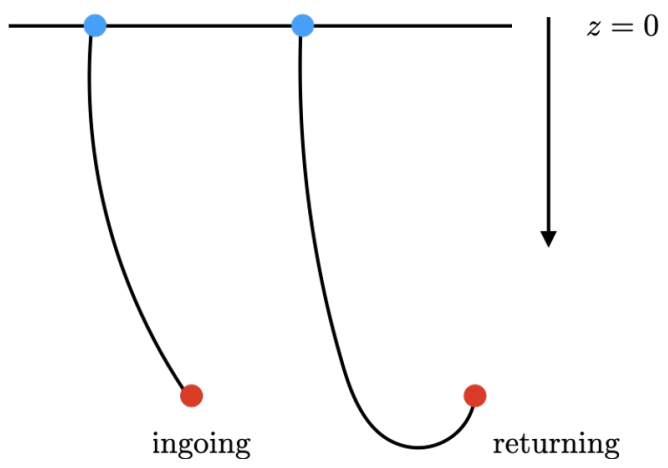

Figure 1. The two types of geodesics to consider.

This can be viewed as a condition that implicitly defines $\mu=\mu\left(\tau_{I}, z_{I}\right)$. The equation (2.10) cannot be inverted explicitly to find an explicit formula for $\mu\left(\tau_{I}, z_{I}\right)$, except in the case of $d=2$. Nevertheless, the implicit definition implied by the condition (2.10) is enough in order to determine the saddle point and thereby the three-point correlation function.

From (2.9), it is clear that the maximum reach inside the bulk of the geodesic, i.e. the turning point where $d z / d \tau=0$, is given by the equation

$$
f\left(z_{\max }\right)=\mu^{2} z_{\max }^{2} .
$$

In (2.10) we assumed that the sign describing the geodesic going towards the black hole. These will be called ingoing geodesics, that is, geodesics departing from the boundary at $x$ and arriving to $u_{I}$ before getting to $z_{\max }$. For the calculation of the three-point function, configurations where geodesics reach $z_{\max }$ and then meet $u_{I}$ in their return way will also be relevant. These will be called returning geodesics. These two types of geodesics are shown in figure 1.

The analysis of each case is carried out in appendix A. The contribution to the action for each type of geodesic is, respectively, given by

$$
S_{\text {ingoing }}=-i \Delta \int_{0}^{z_{i}} d z \frac{1}{z \sqrt{f-\mu^{2} z^{2}}} .
$$

and

$$
S_{\text {returning }}=-i \Delta \int_{0}^{z_{\max }} d z \frac{1}{z \sqrt{f(z)-\mu^{2} z^{2}}}+i \Delta \int_{z_{\max }}^{z_{I}} d z \frac{1}{z \sqrt{f(z)-\mu^{2} z^{2}}} .
$$

As discussed above, here $\mu$ is to be understood as an integration constant, which, through the equation of motion (see appendix A), has to be tuned so that the geodesic arc meets the desired boundary conditions: departing the boundary from the corresponding point $x$ and arriving at the intersection point $u_{I}$.

The total action in (2.7) will be a sum of geodesic arcs, each of them either ingoing — contributing as (2.12) — or returning — contributing as (2.13). The choice of ingoing/returning is determined by the dynamics. 
The formula (2.7) for the three-point function requires to evaluate the $u$ integral through the saddle-point method, which implies an extremization of the total action in the $\tau$ and $z$ direction. Using the formulas of appendix A, we obtain

$$
\begin{aligned}
\frac{d S}{d z_{I}} & =0: & \sum_{i=1}^{3} \epsilon_{i} \Delta_{i} \sqrt{f\left(z_{I}\right)-\mu_{i}^{2} z_{I}^{2}} & =0, \\
\frac{d S}{d \tau_{I}} & =0: & \sum_{i=1}^{3} \Delta_{i} \mu_{i} & =0 .
\end{aligned}
$$

where $\epsilon=1$ for ingoing geodesics and $\epsilon=-1$ for returning geodesics.

\section{Thermal 2-point functions in $d$ dimensions}

The 2-point functions of operators with $\Delta \gg 1$ can be computed in terms of a single geodesic departing from the boundary from a point $x_{1}$, making an excursion into the bulk and coming back to another point $-x_{1}$. One writes

$$
\left\langle O_{\Delta}\left(x_{1}\right) O_{\Delta_{2}}\left(-x_{1}\right)\right\rangle \approx e^{-i S} .
$$

The geodesic is uniquely determined in terms of $x_{1}$. Alternatively, the two-point function can also be computed using the formalism developed above. We view the geodesic as two geodesic arcs joining at some bulk point $u$ (see e.g. [26-28]). Then the two-point function can be computed as the integral

$$
\left\langle O_{\Delta}\left(x_{1}\right) O_{\Delta_{2}}\left(-x_{1}\right)\right\rangle \approx \int d u e^{-i \Delta \ell\left(x_{1}, u\right)} e^{-i \Delta \ell\left(-x_{1}, u\right)} .
$$

The integral can be evaluated by a saddle-point approximation, with the action being $S=\Delta \ell\left(x_{1}, u\right)+\Delta \ell\left(x_{2}, u\right)$. By symmetry, the saddle-point must be located at $\left(\overrightarrow{0}, z_{I}\right)$. Using (2.14), the saddle point equations are

$$
\Delta \mu_{1}+\Delta \mu_{2}=0, \quad \epsilon_{1} \Delta \sqrt{f\left(z_{I}\right)-\mu_{1}^{2} z_{I}^{2}}+\epsilon_{2} \Delta \sqrt{f\left(z_{I}\right)-\mu_{2}^{2} z_{I}^{2}}=0 .
$$

The first condition sets $\mu_{1}=-\mu_{2}=\mu$ (which is nothing but momentum conservation). Since both geodesics are ingoing, $\epsilon_{1}=\epsilon_{2}=1$. Thus we get the condition

$$
f\left(z_{I}\right)-\mu^{2} z_{I}^{2}=0 .
$$

This is precisely the maximum reach for the geodesic, see (2.11), which implies that the arcs are just the arms of a single U-shaped geodesic, as expected, in this way reproducing the result obtained by a direct calculation of the action (3.1).

Let us now consider the explicit calculation of the correlator $\left\langle O_{\Delta}\left(\tau_{1}\right) O_{\Delta}\left(-\tau_{1}\right)\right\rangle$ in $d$ dimensions. It is convenient to solve (3.4) for $\mu$, so that $\mu^{2}=\frac{f\left(z_{I}\right)}{z_{I}^{2}}$. Then, using the formulas from appendix A, we have

$$
\begin{aligned}
S & =-2 i \Delta z_{I} \int_{0}^{z_{I}} d z \frac{1}{z \sqrt{f(z) z_{I}^{2}-f\left(z_{I}\right) u^{2}}} \\
\tau_{I} & =\tau_{1}+\sqrt{f\left(z_{I}\right)} \int_{0}^{z_{I}} d z \frac{z}{f(z) \sqrt{f(z) z_{I}^{2}-f\left(z_{I}\right) z^{2}}}
\end{aligned}
$$


The correlation function is found by substituting into $\exp (-i S)$ the expression of $z_{I}$ in terms of $\tau_{I}$, implicitly defined by (3.5). Since (3.5) cannot be inverted in general, we can work in perturbation theory in the temperature. This is equivalent to a short-distance expansion of the correlator and it amounts to expanding (3.5) in powers of $z^{2}$. We find

$$
z_{I}=\tau_{1}+\frac{\tau_{1}^{d+1}}{4 z_{0}^{d}}\left(\frac{(d-1) \sqrt{\pi} \Gamma\left(1+\frac{d}{2}\right)}{\Gamma\left(\frac{d+3}{2}\right)}-2\right)+\cdots
$$

This gives

$$
\langle O(\tau) O(0)\rangle=\frac{1}{\tau^{2 \Delta}} \exp \left[\alpha_{d} \Delta T^{d} \tau^{d}\left(1+\mathcal{O}\left((T \tau)^{d}\right)\right)\right], \quad \alpha_{d} \equiv \frac{\pi^{d+1} \Gamma(d)}{d^{d} \Gamma\left(\frac{d+3}{2}\right) \Gamma\left(\frac{d-1}{2}\right)} .
$$

where $\tau=\frac{\tau_{1}}{2}$.

\subsection{Universal formula for the free energy}

The thermal 2-point function, in the regime $T|x| \ll 1$, contains interesting information about the $O \times O$ OPE

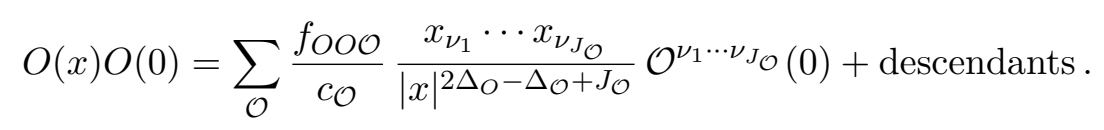

where $c_{\mathcal{O}}$ is the coefficient of the 2-point function of the primaries $\mathcal{O}$ normalized as

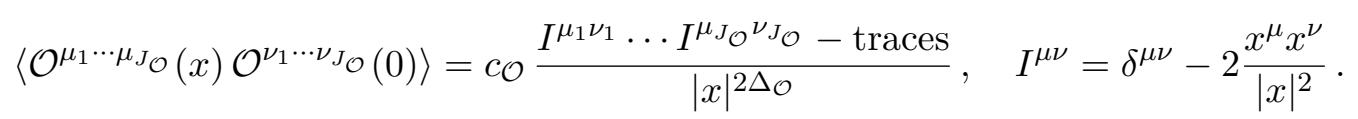

The key point is that the $S^{1} \times \mathbb{R}^{d-1}$ geometry provides, through the circle, both a special direction and a scale allowing for non-zero VEVs of the form,

$$
\left\langle\mathcal{O}^{\mu_{1} \cdots \mu_{J_{\mathcal{O}}}}(x)\right\rangle=\frac{b_{\mathcal{O}}}{\beta^{\Delta_{\mathcal{O}}}}\left(e^{\mu_{1} \cdots \mu_{J_{\mathcal{O}}}}-\text { traces }\right), \quad e^{\mu}=\delta_{\tau}^{\mu} .
$$

Combining with (3.8) and doing the tensor contractions, one finds that, when $T|x| \ll 1$, the thermal 2-point function admits the expansion [1]

$$
\langle O(x) O(0)\rangle=\sum_{\mathcal{O}} \frac{a_{O O \mathcal{O}}}{\beta^{\Delta_{\mathcal{O}}}} \frac{1}{|x|^{2 \Delta_{O}-\Delta_{\mathcal{O}}}} C_{J_{\mathcal{O}}}^{(\nu)}(\eta), \quad a_{O O \mathcal{O}}=\frac{f_{O O \mathcal{O}} b_{\mathcal{O}}}{c_{\mathcal{O}}} \frac{J_{\mathcal{O}} !}{2^{J_{\mathcal{O}}}(\nu)_{J_{\mathcal{O}}}}
$$

where $\eta \equiv \tau /|x|$ and $C_{J_{\mathcal{O}}}^{(\nu)}(\eta)$ are Gegenbauer polynomials $\left(\nu \equiv \frac{d-2}{2}\right)$.

As noted in [3] for the $d=2,4$ cases, the thermal 2-point function (3.7) becomes exact in the double-scaling limit,

$$
\Delta \rightarrow \infty, \quad|x| T \rightarrow 0, \quad \Delta(|x| T)^{d}=\text { fixed } .
$$

In this limit, the $\mathcal{O}\left((T \tau)^{d}\right)$ terms in (3.7) vanish. The full spacetime dependence can now be restored using the consistency with the OPE (3.11). This implies that the exponent 
must be the $\vec{x}=0$ limit of the second Gegenbauer polynomial (this was shown explicitly to be the case in $d=2,4$ in [3]). Therefore we can write

$$
\langle O(\tau) O(0)\rangle=\frac{1}{\tau^{2 \Delta}} \exp \left[A_{d} \Delta T^{d}|x|^{d} C_{2}^{\left(\frac{d-2}{2}\right)}(\eta)\right], \quad A_{d} \equiv \frac{2^{d-2} \pi^{\frac{1+2 d}{2}} \Gamma\left(\frac{d-2}{2}\right)}{d^{d} \Gamma\left(\frac{d+3}{2}\right)} .
$$

Let us consider explicitly the case of $d=4$. Expanding the exponential, one finds

$$
\left\langle O_{\Delta}(x) O_{\Delta}(0)\right\rangle=\sum_{J=0} \sum_{n=0} \frac{1}{n !}\left(\frac{\pi^{4} \Delta}{120}\right)^{n} \frac{\mathcal{C}_{J, n}}{\beta^{4 n}} \frac{1}{|x|^{2 \Delta-4 n}} C_{J}^{(1)}(\eta),
$$

where the numerical coefficients $\mathcal{C}_{J, n}$ turn out to be given as well in terms of Gegenbauer polynomials as

$$
\mathcal{C}_{J, n}=\frac{2}{\pi} \int_{-1}^{1} d \eta \sqrt{1-\eta^{2}} C_{J}^{(1)}(\eta)\left(C_{2}^{(1)}(\eta)\right)^{n}= \begin{cases}C_{n-\frac{J}{2}}^{(-n)}\left(-\frac{1}{2}\right)-C_{n-\frac{J}{2}-1}^{(-n)}\left(-\frac{1}{2}\right), & \text { if } n, J \neq 0 \\ 1 & \text { if } n, J=0\end{cases}
$$

Comparing with the general form of the correlator, each term in the series in (3.13) represents the contribution of a spin $J$ and dimension $4 n$ operator constructed as the appropriate contraction of $n$ factors of energy-momentum tensors, which we will denote schematically as $\mathcal{T}^{n}$. Clearly, the spin of such contraction is at most $2 n$. Consistently, the coefficients in (3.15) vanish if $J>2 n$. Moreover, the contribution of each term scales with $\Delta^{n}=\Delta^{\frac{\Delta_{\mathcal{T}}}{4}}$, which is precisely the large $\Delta$ scaling expected [5] (see also [29]). Note that this extends to arbitrary dimensions, as the expansion of (3.13) involves $\Delta^{n}=\Delta^{\frac{d n}{d}}=\Delta^{\frac{\Delta_{\mathcal{T} n}}{d}}$.

The leading term in the expansion of (3.13) corresponds to the $J=2$ term in (3.11). This yields the identification $a_{\mathcal{T}}=A_{d}$. The OPE coefficient $f_{O O \mathcal{T}}$ with the energymomentum tensor is fixed by a Ward identity. One has [1]

$$
f_{O O \mathcal{T}}=-\frac{d}{d-1} \frac{\Delta}{\operatorname{vol}\left(S^{d-1}\right)}
$$

Thus, combining with (3.11), we find a universal formula for the vacuum expectation value for the energy density

$$
\frac{\left\langle\mathcal{T}^{00}\right\rangle_{\beta}}{c_{\mathcal{T}}}=-\frac{2^{2 d-1}(d-1)^{2} \pi^{\frac{3 d}{2}} \Gamma\left(\frac{d}{2}\right)}{d^{d} \Gamma(d+2)} T^{d}
$$

This formula can be alternatively derived from the thermodynamics of black holes supplemented with the AdS/CFT dictionary relating the Newton constant and $c_{\mathcal{T}}$ as in e.g. [30].

Using the standard thermodynamic relation, $F=E+T \frac{d F}{d T}$, we find

$$
F=-\frac{2^{2 d-1}(d-1) \pi^{\frac{3 d}{2}} \Gamma\left(\frac{d}{2}\right)}{d^{d} \Gamma(d+2)} c_{\mathcal{T}} T^{d}
$$


We can now combine this formula with the general expression for the free energy given in [22] (see also [31]) to provide an independent derivation of the central charge for ABJM, $\mathcal{N}=4 \mathrm{SYM}$ and the $6 \mathrm{~d}(2,0)$ theory for $d=3,4,6$ respectively. We find

$$
c_{\mathcal{T}}^{\mathrm{ABJM}}=\frac{2 \sqrt{2}}{\pi^{3}} N^{\frac{3}{2}}, \quad c_{\mathcal{T}}^{\mathcal{N}}=4=\frac{10}{\pi^{2}} N^{2}, \quad c_{\mathcal{T}}^{(2,0)}=4 N^{3} \frac{84}{\pi^{6}} .
$$

Note that these central charges are in the convention where a free real scalar contributes as

$$
c_{\mathcal{T}}^{\text {free scalar }}=\frac{d}{d-1} \frac{1}{S_{d}^{2}}, \quad S_{d}=\frac{2 \pi^{\frac{d}{2}}}{\Gamma\left(\frac{d}{2}\right)} .
$$

For $\mathcal{N}=4$ SYM, using [23] with $n_{\phi}=6$ real scalars, $n_{\psi}=\frac{4}{2}$ Dirac fermions and one gauge field in $d=4$, one precisely recovers the expression of $c_{\mathcal{T}}^{\mathcal{N}}=4$ given above.

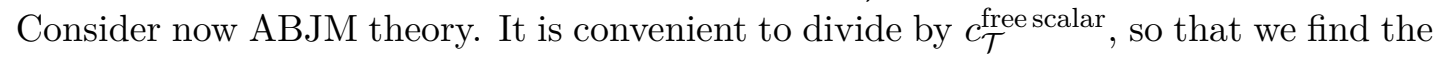
central charge in the conventions where a free real scalars contributes 1 - we will denote it with a hat. We find

$$
\hat{c}_{\mathcal{T}}^{\mathrm{ABJM}}=\frac{64 \sqrt{2}}{3 \pi} N^{\frac{3}{2}}
$$

in agreement with [25] for $k=1$ (see eq. (5.11)) (see also [24]).

Finally, for the $6 \mathrm{~d}(2,0)$ theory, note that in our conventions, a free tensor multiplet contributes as [24] (see eqs. (2.6) and (3.23))

$$
c_{\mathcal{T}}^{\text {free } 6 \mathrm{~d} \text { tensor }}=\frac{84}{\pi^{6}}
$$

Thus, in units where the free tensor multiplet contributes 1 , we have

$$
\hat{c}_{\mathcal{T}}^{(2,0)}=4 N^{3},
$$

reproducing the known result.

\subsection{Including $\alpha^{\prime}$ corrections in the $d=4$ case}

One of the famous problems in AdS/CFT concerns the match between the holographic free energy and the free energy derived from $\mathcal{N}=4$ super Yang-Mills theory. As known, the weak-coupling free energy differs from the strong-coupling (holographic) result by a factor of $3 / 4$. It is believed that both limits arises from a monotonic function $f(\lambda)$, which interpolates between 1 and $3 / 4$. The leading strong-coupling correction to $f(\lambda)$ was computed in [22] by computing the explicit modifications to the black brane geometry induced by the $\alpha^{\prime 3} R^{4}$ corrections to the type IIB effective action. In the present formalism, we may similarly include corrections by considering the geodesic moving in the $\alpha^{\prime}$ corrected background. We will ignore other possible sources of $\alpha^{\prime}$ corrections, such as possible higherderivative interactions of the bulk scalar field, assuming that the leading-order correction comes from the $\alpha^{\prime}$ corrected background.

The first observation is that $\alpha^{\prime}$ corrections arise in powers of $\frac{z^{4}}{z_{0}^{4}}$. Thus the net effect of the $\alpha^{\prime}$ corrections will be to change the $a_{O O \mathcal{O}}$ coefficients, as opposed to turning on new 
terms (for instance, a term proportional to $C_{0}^{(1)}$ corresponding to a dimension 2 scalar is not induced, as it would require a $\frac{z^{2}}{z_{0}^{2}}$ correction). As a result of corrections to the background, the relation between $z_{0}$ and the temperature also gets $\alpha^{\prime}$ corrections. It becomes

$$
z_{0}=\frac{1+15 \gamma}{\pi T}, \quad \gamma=\frac{1}{8} \zeta(3)\left(\alpha^{\prime}\right)^{3},
$$

in units where the AdS radius is equal to 1 . The correction to the energy density $\left\langle\mathcal{T}^{00}\right\rangle_{\beta}$ shows up in the coefficient of the term $T^{4}$ in the expansion of the exponent in (3.13). This is affected by the leading $\frac{z^{4}}{z_{0}^{4}}$ term in the expansion. Therefore, the leading $\alpha^{\prime}$ correction to the energy density can be obtained from the $O\left(z^{4}\right)$ correction in the metric. Formally, one gets the same metric as in the unperturbed case, by trading $z_{0}^{4}$ by $z_{0, \text { corrected }}^{4}(1+75 \gamma)^{-1}$, where we have included the subscript to emphasize that this is the $\alpha^{\prime}$ corrected $z_{0}$ given in (3.24). Using (3.24), we obtain the re-scaling,

$$
z_{0}=\frac{1}{\pi T} \rightarrow \frac{(1+15 \gamma)(1+75 \gamma)^{-\frac{1}{4}}}{\pi T} \sim \frac{1}{\pi T(1+15 \gamma)^{\frac{1}{4}}}
$$

Thus,

$$
T^{4} \rightarrow T^{4}(1+15 \gamma)=T^{4}\left(1+\frac{15}{8} \zeta(3)\left(2 g_{\mathrm{YM}}^{2} N\right)^{-\frac{3}{2}}\right)
$$

This leads to the free energy

$$
F=-\frac{\pi^{2}}{6} N^{2} T^{4}\left(\frac{3}{4}+\frac{45}{32} \zeta(3)\left(2 g_{\mathrm{YM}}^{2} N\right)^{-\frac{3}{2}}+\cdots\right)
$$

This is precisely the leading correction at strong coupling found in [22].

\section{Thermal 3-point correlation functions with reflection symmetry}

Let us now consider thermal 3-point functions at coincident spatial points. According to the previous discussion, this is computed holographically as

$$
\left\langle O_{\Delta_{1}}\left(\tau_{1}\right) O_{\Delta_{2}}\left(\tau_{1}\right) O_{\Delta_{3}}\left(\tau_{3}\right)\right\rangle \sim e^{-i S}
$$

where $S=\sum_{i=1,2,3} S_{\Delta_{i}}\left(\tau_{i}, u_{I}\right)$, being $S_{\Delta_{i}}\left(\tau_{i}, u_{I}\right)$ the contribution of an ingoing/returning geodesics from $\tau_{i}$ at the boundary to the intersection point $u_{I}$ - to be fixed through extremization of $S$.

With no loss of generality, one can set $\tau_{3}=0$. Then the relevant geodesic configuration is dynamically fixed and it depends on the values of $\tau_{1}, \tau_{2}$ and $\Delta_{1,2,3}$ (more specifically, on two ratios, e.g. $\left.\Delta_{1} / \Delta_{3}, \Delta_{2} / \Delta_{3}\right)$. The key equation is

$$
\epsilon_{1} \Delta_{1} \sqrt{f\left(z_{I}\right)-\mu_{1}^{2} z_{I}^{2}}+\epsilon_{2} \Delta_{2} \sqrt{f\left(z_{I}\right)-\mu_{2}^{2} z_{I}^{2}}+\epsilon_{3} \Delta_{3} \sqrt{f\left(z_{I}\right)-\mu_{3}^{2} z_{I}^{2}}=0,
$$

with $\Delta_{1} \mu_{1}+\Delta \mu_{2}+\Delta_{3} \mu_{3}=0$. For given values of the parameters, this equation will have a unique real solution $z_{I} \in(0,1)$, for a specific choice of signs $\epsilon_{1,2,3}$ (and other choices of sign will give no real solution $\left.z_{I} \in(0,1)\right)$. This determines which geodesics are ingoing 


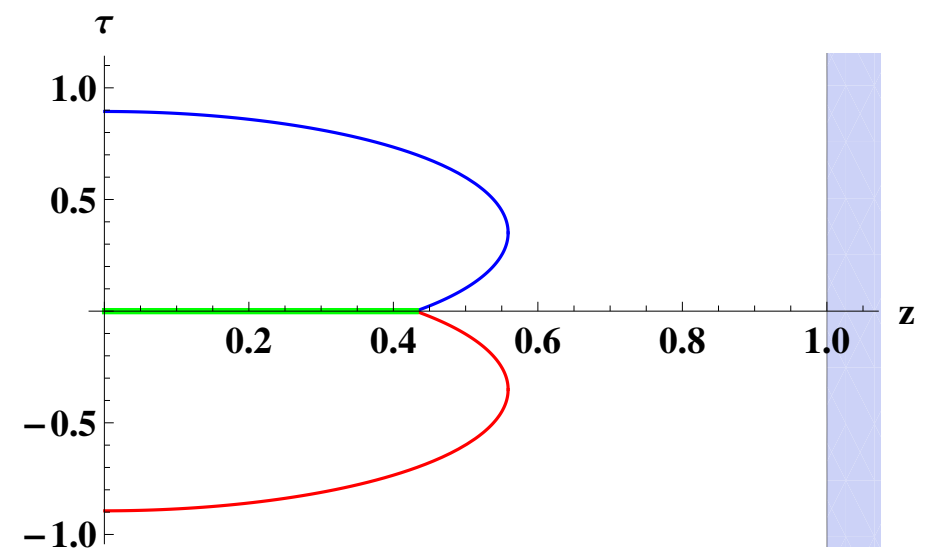

Figure 2. A symmetric configuration for $\Delta_{1}=\Delta_{2}=30$ and $\Delta_{3}=40$, with the operator $O_{3}$ placed in the middle point between $O_{1}$ and $O_{2}$. Geodesics 1,2 are returning and geodesic 3 is ingoing. In this symmetric case, the latter is a straight line between $z=0$ and $z_{I}$. [Red, blue and green colors represent the geodesics representing operators $\mathrm{O}_{1}, \mathrm{O}_{2}$ and $\mathrm{O}_{3}$, respectively].

and which ones are returning. For example, for $\tau_{1}$ sufficiently close to $\tau_{2}$, the geodesics for operators 1 and 2 must be ingoing and the geodesic 3 must be returning. On the other hand, if we choose $\tau_{3}=0, \tau_{1}$ and $\tau_{2}$ sufficiently separated and $\Delta_{3}<\Delta_{1}+\Delta_{2}$, geodesics 1 and 2 are returning and 3 is outgoing. This will be illustrated by several examples in the following sections.

We begin with a case where calculations dramatically simplify. This is the case of a "symmetric" correlator $\left\langle O_{\Delta}(\tau) O_{\Delta^{\prime}}(0) O_{\Delta}(-\tau)\right\rangle$. In this case, the only consistent solution to the saddle-point equation implies that $O_{\Delta}(\tau)$ and $O_{\Delta}(-\tau)$ correspond to returning geodesics while $O_{\Delta^{\prime}}(0)$ corresponds to an ingoing geodesic (see figure 2). Then

$$
\begin{aligned}
& \left\langle O_{\Delta}(\tau) O_{\Delta^{\prime}}(0) O_{\Delta}(-\tau)\right\rangle \approx e^{-i S}, \\
& S=S_{\Delta}^{\text {returning }}\left(-\tau_{1}, u_{I}\right)+S_{\Delta}^{\text {returning }}\left(\tau_{1}, u_{I}\right)+S_{\Delta^{\prime}}^{\text {ingoing }}\left(0, u_{I}\right)
\end{aligned}
$$

By symmetry, $\tau_{I}=0$, therefore the ingoing geodesic corresponding to $O_{\Delta^{\prime}}(0)$ is actually a straight line at constant $\tau=0$ that extends from the boundary up to $z_{I}$. This fixes the corresponding $\mu^{\prime}=0$, and, through the second equation in (2.14), sets $\mu_{1}=-\mu_{2} \equiv \mu$. Therefore, we are left with the first equation, which becomes

$$
2 \Delta \sqrt{f\left(z_{I}\right)-\mu^{2} z_{I}^{2}}-\Delta^{\prime} \sqrt{f\left(z_{I}\right)}=0 .
$$

\subsection{Symmetric three-point function in $d=2$}

As a warm-up, we start considering the symmetric thermal three-point function in the simpler $d=2$ case. We consider the configuration of figure 2 . In two dimensions, the integral defining the geodesic $\tau(z, \mu)$ gives simple logarithmic functions, which permits to find an explicit closed formula for $\mu$ in terms of the boundary coordinate $\tau$. The saddle- 
point equation for $z$ is solved by

$$
z_{I}=z_{\max } \frac{\sqrt{4 \Delta^{2}-\Delta^{\prime 2}}}{\sqrt{4 \Delta^{2}-z_{\max }^{2} \Delta^{\prime 2}}}, \quad z_{\max }=\frac{z_{0}}{\sqrt{1+z_{0}^{2} \mu^{2}}} .
$$

Substituting into the equation for the geodesic, at the intersection point we have $0=$ $\tau\left(z_{I}, \mu\right)$. This can be explicitly solved for $\mu$. We find $\left(z_{0}=1\right)$

$$
\mu=\cot \tau+\frac{\Delta^{\prime}}{2 \Delta \sin \tau} .
$$

Substituting into the action, we obtain

$$
\left\langle O_{\Delta}(\tau) O_{\Delta^{\prime}}(0) O_{\Delta}(-\tau)\right\rangle=\left(1-\frac{\Delta^{\prime}}{2 \Delta}\right) \Delta-\frac{\Delta^{\prime}}{2}\left(1+\frac{\Delta^{\prime}}{2 \Delta}\right) \Delta+\frac{\Delta^{\prime}}{2} \frac{\cot ^{\Delta^{\prime}}(\pi T \tau)}{\sin ^{2 \Delta}(2 \pi T \tau)},
$$

where the temperature has been restored, recalling that $z_{0}=1 /(2 \pi T)$. This agrees with the result of [32]. Note that, in the "extremal" limit $\Delta_{0} \rightarrow 2 \Delta$, one obtains ${ }^{2}$

$$
\left\langle O_{\Delta}(\tau) O_{2 \Delta}(0) O_{\Delta}(-\tau)\right\rangle=\frac{1}{(\sin (\pi T \tau))^{4 \Delta}},
$$

which is, up to a $\Delta$-dependent constant, the product of two 2-point functions,

$$
\left\langle O_{\Delta}(\tau) O_{2 \Delta}(0) O_{\Delta}(-\tau)\right\rangle=\mathcal{N}_{\Delta}\left\langle O_{\Delta}(\tau) O_{\Delta}(0)\right\rangle\left\langle O_{\Delta}(0) O_{\Delta}(-\tau)\right\rangle .
$$

We will return to this factorization property in section 5 .

Beyond the extremal limit, when $\Delta^{\prime}>2 \Delta$, the saddle point no longer lies within the region $0<z<z_{0}$. The correlation function can be defined by analytic continuation and it is of the same form (4.6) (for $2 \Delta<\Delta^{\prime}<2 \Delta / z_{\max }$, the saddle point is imaginary; for $\Delta^{\prime}>2 \Delta / z_{\max }$, it lies inside the horizon).

\subsection{Symmetric three-point function in $d=4$}

In $d=4$ the solution to (4.4) is

$$
z_{I}=\sqrt{\frac{\sqrt{4\left(1-\delta^{2}\right)^{2}+\mu^{2}}-\mu^{2}}{2\left(1-\delta^{2}\right)}}, \quad \delta=\frac{\Delta^{\prime}}{2 \Delta} .
$$

Here we have chosen units where $1=z_{0}=\beta / \pi$. We assume $\delta<1$, that is, the "nonextremal case' $\Delta^{\prime}<2 \Delta$. As in the $d=2$ case, for $\Delta^{\prime}>2 \Delta$ the correlator is defined by analytic continuation.

Substituting (4.9) into the geodesic equation $\tau=\tau(z, \mu)$, we find the condition at the intersection point, $0=\tau\left(z_{I}, \mu\right)$. This can be solved for $\mu$ in series of small $\tau_{1}$. We get

$$
\mu=\frac{1+\frac{\Delta^{\prime}}{2 \Delta}}{\tau_{1}+\frac{32 \Delta^{2}-18 \Delta \Delta^{\prime}+3 \Delta^{\prime 2}}{10\left(2 \Delta+\Delta^{\prime}\right)^{2}} \tau_{1}^{5}+\cdots} .
$$

\footnotetext{
${ }^{2}$ By extremal correlators we mean those where the dimension of one operator equals the sum of the other two.
} 
Substituting this into $S$ one finally finds

$$
\begin{aligned}
\left\langle O_{\Delta}\left(\tau_{1}\right) O_{\Delta^{\prime}}(0) O_{\Delta}\left(-\tau_{1}\right)\right\rangle & =\frac{e^{-i S_{T}}}{\tau_{1}^{2 \Delta+\Delta^{\prime}}} \\
-i S_{T} & =\frac{\left(32 \Delta^{2}-18 \Delta \Delta^{\prime}+3 \Delta^{\prime 2}\right)}{40\left(2 \Delta+\Delta^{\prime}\right)} \pi^{4} T^{4} \tau_{1}^{4}+\mathcal{O}\left(T^{8} \tau_{1}^{8}\right) .
\end{aligned}
$$

where the temperature has been restored. As a sanity check, in the $T=0$ limit we find $\left\langle O_{\Delta}\left(\tau_{1}\right) O_{\Delta^{\prime}}(0) O_{\Delta}\left(-\tau_{1}\right)\right\rangle=\tau_{1}^{-\left(2 \Delta+\Delta^{\prime}\right)}$, which is precisely the expected result (cf. (5.1)).

Let us now consider different limits. For the hierarchy $\Delta \gg \Delta^{\prime}$, to leading order we have

$$
\left\langle O_{\Delta}\left(-\tau_{1}\right) O_{\Delta^{\prime}}(0) O_{\Delta}\left(-\tau_{1}\right)\right\rangle \approx \frac{e^{\frac{\pi^{4}}{40} \Delta T^{4} \tau^{4}}}{\tau^{2 \Delta+\Delta^{\prime}}},
$$

where $\tau=2 \tau_{1}$. This approaches the 2-point function $\left\langle O_{\Delta}\left(\tau_{1}\right) O_{\Delta}\left(-\tau_{1}\right)\right\rangle$.

On the other hand, in the 'extremal' limit $\Delta^{\prime}=2 \Delta$, we find

$$
\left\langle O_{\Delta}\left(\tau_{1}\right) O_{2 \Delta}(0) O_{\Delta}\left(-\tau_{1}\right)\right\rangle \rightarrow\left(\frac{e^{\frac{\pi^{4} \Delta T^{4} \tau_{1}^{4}}{40}}}{\tau_{1}^{2 \Delta}}\right)^{2}=\mathcal{N}_{\Delta}\left\langle O_{\Delta}\left(\tau_{1}\right) O_{\Delta}(0)\right\rangle\left\langle O_{\Delta}(0) O_{\Delta}\left(-\tau_{1}\right)\right\rangle
$$

that is, the extremal 3-point function again factorizes as the product of two 2-point functions.

\section{General three-point correlation function and factorization limit}

\subsection{Factorization}

In the previous section, we considered the symmetric 3-point function $\left\langle O_{\Delta}\left(\tau_{1}\right) O_{\Delta^{\prime}}(0)\right.$ $\left.O_{\Delta}\left(-\tau_{1}\right)\right\rangle$ in $d=2,4$. The symmetric configuration makes it possible to find an analytic formula, which is exact in the $d=2$ case, and in the $d=4$ case it describes a $T|\tau| \ll 1$ regime. Both in $d=2,4$ the three-point function satisfies a surprising factorization property in the "extremal" limit $\Delta^{\prime} \rightarrow 2 \Delta$. In this subsection we will clarify the origin of this factorization and study it for general three-point functions.

At $T=0$, the factorization property is manifest from the generic form of the 3-point function, which is entirely fixed by conformal symmetry,

$$
\left\langle O_{\Delta_{1}}\left(x_{1}\right) O_{\Delta_{2}}\left(x_{2}\right) O_{\Delta_{3}}\left(x_{3}\right)\right\rangle=\frac{f_{O_{\Delta_{1}} O_{\Delta_{2}} O_{\Delta_{3}}}}{\left|x_{1}-x_{2}\right|^{\Delta_{1}+\Delta_{2}-\Delta_{3}}\left|x_{2}-x_{3}\right|^{\Delta_{2}+\Delta_{3}-\Delta_{1}}\left|x_{3}-x_{1}\right|^{\Delta_{3}+\Delta_{1}-\Delta_{2}}} .
$$

Setting, for example, $\Delta_{3}=\Delta_{1}+\Delta_{2}$, one gets

$$
\begin{aligned}
\left\langle O_{\Delta_{1}}\left(x_{1}\right) O_{\Delta_{2}}\left(x_{2}\right) O_{\Delta_{1}+\Delta_{2}}\left(x_{3}\right)\right\rangle & \sim \frac{1}{\left|x_{1}-x_{3}\right|^{2 \Delta_{1}}\left|x_{2}-x_{3}\right|^{2 \Delta_{2}}} \\
& \sim\left\langle O_{\Delta_{1}}\left(x_{1}\right) O_{\Delta_{1}}\left(x_{3}\right)\right\rangle\left\langle O_{\Delta_{2}}\left(x_{2}\right) O_{\Delta_{2}}\left(x_{3}\right)\right\rangle .
\end{aligned}
$$

However, at $T \neq 0$ the form of the 3-point function is not fixed by symmetries and it has an extremely complicated general structure. 
To start with, let us first consider a generic three-point correlation function with only time-dependence. With no loss of generality, we can place one of the operators at $\tau=0$. Thus we consider correlators of the form $\left\langle O_{\Delta_{1}}\left(-\tau_{1}\right) O_{\Delta_{3}}(0) O_{\Delta_{2}}\left(\tau_{2}\right)\right\rangle$ and, for concreteness, we will initially assume that with $\tau_{1}>0$ and $\tau_{2}>0$. For large dimensions, $\Delta_{i} \gg 1$, the correlation function is represented by three geodesic arcs meeting at some point $\left(\tau_{I}, z_{I}\right)$ in the bulk. An examination of the saddle-point equation shows that, for $\Delta_{3}<\Delta_{1}+\Delta_{2}$, and $\Delta_{3}$ sufficiently close to $\Delta_{1}+\Delta_{2}$, the operator $O_{\Delta_{3}}$ is described by an ingoing geodesic while $O_{\Delta_{1,2}}$ are described by returning geodesics, generalizing the picture of figure 2 . The saddle point equations then read

$$
\begin{aligned}
\Delta_{1} \sqrt{f\left(z_{I}\right)-\mu_{1}^{2} z_{I}^{2}}+\Delta_{2} \sqrt{f\left(z_{I}\right)-\mu_{2}^{2} z_{I}^{2}}-\Delta_{3} \sqrt{f\left(z_{I}\right)-\mu_{3}^{2} z_{I}^{2}}=0, \\
\Delta_{1} \mu_{1}+\Delta_{2} \mu_{2}+\Delta_{3} \mu_{3}=0 .
\end{aligned}
$$

Other choices of relative signs do not have real solution $0<z_{I}<1$ in the region $\Delta_{3}=$ $\Delta_{1}+\Delta_{2}-\epsilon$. The saddle-point equation (5.2) can be solved explicitly in terms of one of the roots of the quartic polynomial for $X \equiv z^{2}$, obtained by squaring the saddle-point equation. For sufficiently small $\tau_{1}$ and $\tau_{2}$, as compared with the scale of the thermal circle $\beta$, there is a unique root that represents the dominant saddle point and describes the expected short-distance behavior of the correlation function.

In the region $\Delta_{3} \sim \Delta_{1}+\Delta_{2}$, it has the behavior

$$
z_{I}^{2} \approx \frac{2}{\Delta_{1} \mu_{1}^{2}+\Delta_{2} \mu_{2}^{2}-\left(\Delta_{1}+\Delta_{2}\right) \mu_{3}^{2}}\left(\Delta_{1}+\Delta_{2}-\Delta_{3}\right) \approx 0
$$

Note that $z_{I}$ is real for $\Delta_{3} \leq \Delta_{1}+\Delta_{2}$, and imaginary for $\Delta_{3}>\Delta_{1}+\Delta_{2}$.

At the critical point $\Delta_{3}=\Delta_{1}+\Delta_{2}$, we have $z_{I}=0$, and the three point correlation function exhibits the remarkable factorization property:

$$
\begin{aligned}
\left\langle O_{\Delta_{1}}\left(-\tau_{1}\right) O_{\Delta_{3}}(0) O_{\Delta_{2}}\left(\tau_{2}\right)\right\rangle & =\left\langle O_{\Delta_{1}}\left(-\tau_{1}\right) O_{\Delta_{1}}(0)\right\rangle\left\langle O_{\Delta_{2}}(0) O_{\Delta_{2}}\left(\tau_{2}\right)\right\rangle \\
& \approx 4^{-\Delta_{1}}\left(\mu_{1}^{2}+4\right)^{\frac{\Delta_{1}}{2}} 4^{-\Delta_{2}}\left(\mu_{2}^{2}+4\right)^{\frac{\Delta_{2}}{2}}
\end{aligned}
$$

where $\mu_{k}\left(\tau_{k}\right), k=1,2$, are implicitly defined by

$$
\tau=-\frac{1}{2} \log \frac{\mu(\mu-2)+2}{\sqrt{\mu^{2}+4}}+\frac{i}{2} \log \frac{\sqrt{\mu^{2}+4}}{\mu(\mu+2 i)-2} .
$$

The factorization limit is illustrated in figures 3 and 4 . One can see that the geodesic describing the operator $O_{\Delta_{3}}$ shrinks to zero as $\Delta_{3} \sim \Delta_{1}+\Delta_{2}$. In this limit, the configuration reduces to two individual geodesics ending at the same point $\tau_{3}=0$, which represent the two propagators $\left\langle O_{\Delta_{1}}\left(-\tau_{1}\right) O_{\Delta_{1}}(0)\right\rangle$ and $\left\langle O_{\Delta_{2}}(0) O_{\Delta_{2}}\left(\tau_{2}\right)\right\rangle$. 

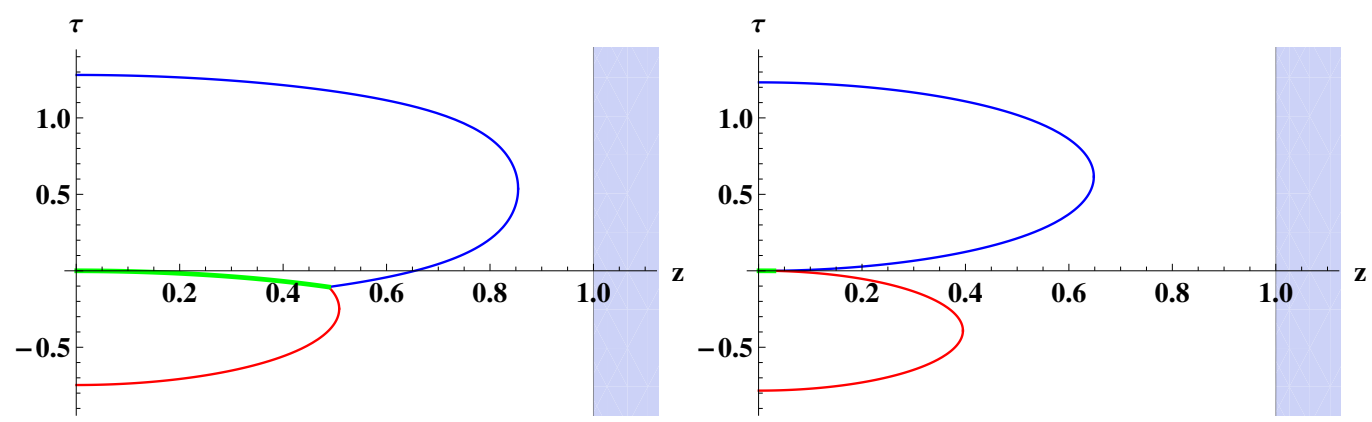

Figure 3. a) Non-symmetric configuration for $\Delta_{1}=\Delta_{2}=30$ and $\Delta_{3}=40$, with the operator $O_{\Delta_{3}}$ placed between $O_{\Delta_{1}}$ and $O_{\Delta_{2}}$. b) As $\Delta_{3} \rightarrow \Delta_{1}+\Delta_{2}$, the configuration reaches the factorization limit. In the figure b), $\Delta_{1}=\Delta_{2}=30$ and $\Delta_{3}=59.9$. [Same color notation as in figure 2.]
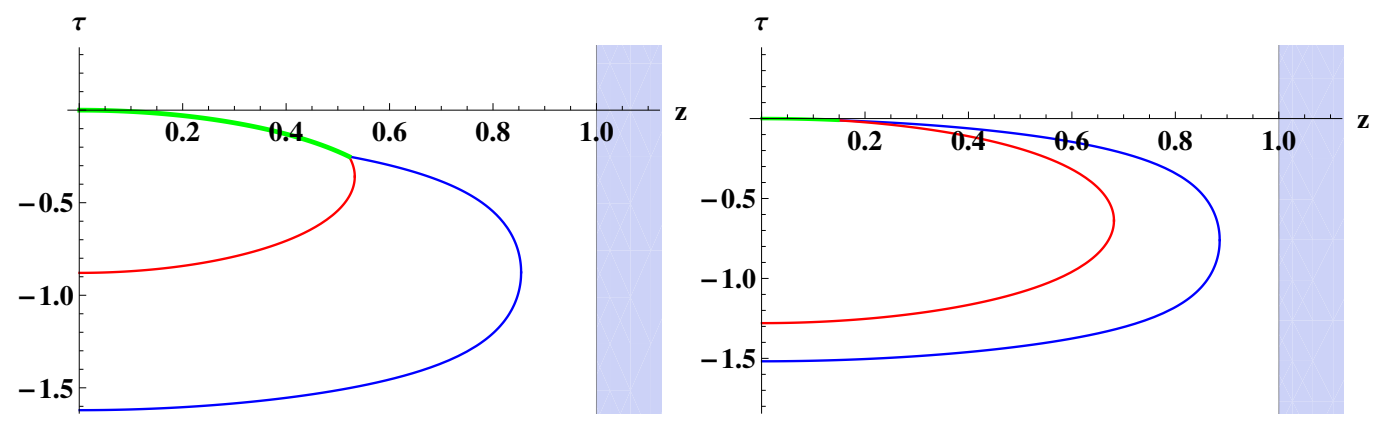

Figure 4. a) Non-symmetric configuration for $\Delta_{1}=50, \Delta_{2}=50$ and $\Delta_{3}=90$, with the operator $O_{\Delta_{1}}$ placed between $O_{\Delta_{2}}$ and $O_{\Delta_{3}}$. b) $\Delta_{1}=50, \Delta_{2}=50$ and $\Delta_{3}=99.9$. [Same color notation as in figure 2.]

\section{$5.2 \quad$ Adding $\vec{x}$ dependence}

Let us now incorporate the $\vec{x}$ dependence. The details are given in appendix A. In the general case, we find the following saddle-point equations

$$
\begin{aligned}
\sum_{i=1}^{3} \epsilon_{i} \Delta_{i} \sqrt{f\left(z_{I}\right)-\mu_{i}^{2} z_{I}^{2}+f\left(z_{I}\right) \nu_{i}^{2} z_{I}^{2}} & =0, \\
\Delta_{1} \mu_{1}+\Delta_{2} \mu_{2}+\Delta_{3} \mu_{3} & =0, \\
\Delta_{1} \nu_{1}+\Delta_{2} \nu_{2}+\Delta_{3} \nu_{3} & =0 .
\end{aligned}
$$

where, again $\epsilon_{i}= \pm 1$ according to whether the corresponding geodesic is ingoing or returning at the point $z_{I}$.

Let us assume that $O_{1}\left(\tau_{1}, \vec{x}_{1}\right)$ and $O_{2}\left(\tau_{2}, \vec{x}_{2}\right)$ are represented by the returning geodesics, and $O_{3}\left(\tau_{3}, \vec{x}_{3}\right)$ by an ingoing geodesic. The factorization limit then occurs when $z_{I} \rightarrow 0$. The saddle-point equation for $z_{I}$ then requires

$$
\sum_{i=1}^{3} \epsilon_{i} \Delta_{i}=\Delta_{3}-\Delta_{1}-\Delta_{2} \rightarrow 0 .
$$



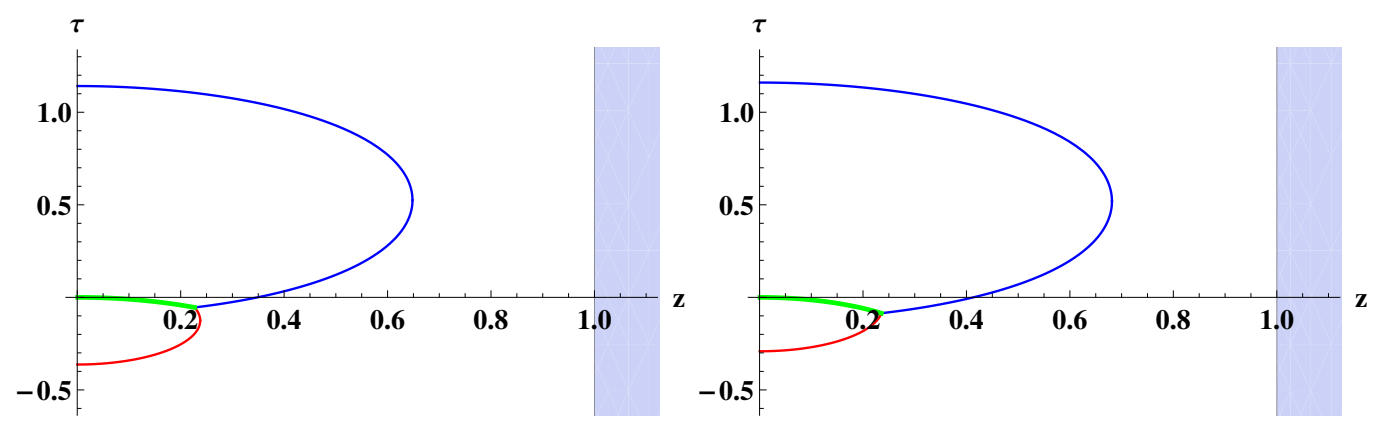

Figure 5. a) Geodesic configuration for $\Delta_{1}=\Delta_{2}=30, \Delta_{3}=42, \tau_{2}=1.15, \tau_{1}=-0.37$. b) Here $\Delta_{1}=\Delta_{2}=30, \Delta_{3}=32, \tau_{2}=1.15, \tau_{1}=-0.3$. The geodesic 1 (in red), which was returning in figure a), is now ingoing. [Same color notation as in figure 2.]

In such a case the ingoing geodesic shrinks to zero and one has

$$
\left\langle O_{\Delta_{1}}\left(x_{1}\right) O_{\Delta_{2}}\left(x_{2}\right) O_{\Delta_{1}+\Delta_{2}}\left(x_{3}\right)\right\rangle \rightarrow \mathcal{N}_{\Delta_{1}, \Delta_{2}}\left\langle O_{\Delta_{1}}\left(x_{1}\right) O_{\Delta_{1}}\left(x_{3}\right)\right\rangle\left\langle O_{\Delta_{2}}\left(x_{2}\right) O_{\Delta_{2}}\left(x_{3}\right)\right\rangle ;
$$

Thus the factorization of the three-point function seems to be a general property, which occurs in the limit $\sum_{i=1}^{3} \epsilon_{i} \Delta_{i} \rightarrow 0$.

As the location points of the operators move around in the boundary, there can be transitions where a returning geodesic becomes ingoing, and vice-versa (see figure 5). A critical point occurs whenever the intersection point coincides with one of the $z_{\max }$ of the three geodesics. A simple examination shows that this never happens in the symmetric case of section 4 . For example, the condition, $z_{\max }^{(1)}=z_{I}$ determines the critical value

$$
\mu_{1}^{c}=-\frac{2 \Delta_{1} \Delta_{2}}{\Delta_{1}^{2}+\Delta_{2}^{2}-\Delta_{3}^{2}} \mu_{2}
$$

Assuming $\mu_{2}<0$, the geodesic 1 is returning for $\mu_{1}<\mu_{1}^{c}$ and ingoing for $\mu_{1}>\mu_{1}^{c}$. For the symmetric case $\mu_{1}=-\mu_{2}, \Delta_{1}=\Delta_{2}$, and it is not possible to have a transition (geodesic 1 is always returning).

It would be very interesting to study this phenomenon in more detail, in particular, the analytic properties of such transitions in the infinite $\Delta_{i}$ limit. On general grounds, one expects that the three-point function should be analytic in $x_{1}, x_{2}, x_{3}$ away from the coinciding points. This is also supported by the fact that ingoing-returning transitions also take place at $T=0$, with the same critical value $\mu_{1}^{c}$. In the $T=0$ case, the well-known 3-point function has a simple form, as being completely fixed by conformal invariance. Clearly, it is an analytic function of $x_{1,2,3}$ away from the coinciding points.

In addition to ingoing-returning transitions, as the separation of the operators along the thermal circle increases and the geodesics go further into the bulk, other saddle points have to be considered. This is analogous to what happens for 2-point functions in $[3,14]$. Although in this paper we restricted the considerations to distances less than the critical distance corresponding to possible saddle-point jumps, it would clearly be very interesting to study these aspects in detail. 


\subsection{Factorization property as a sign of a semiclassical limit}

At $T=0$, extremal correlators have been extensively discussed, albeit mostly in connection with subtleties related to their prefactors: in top-down constructions from type IIB string theory [33], the overall coefficient typically vanishes while at the same time the coupling constant diverges in such a way that these effects cancel leaving a finite result [34, 35]. As far as their spacetime dependence is concerned, because their form is completely fixed by conformal symmetry, factorization arises as a trivial consequence. It is striking that the factorization property of extremal correlators still seems to hold in the general $T \neq 0$ theory, where three-point functions, as well as two-point functions, have a much more complicated structure.

To investigate the implications of this property, let us suppose that we start with a general CFT and take, as a fact, that extremal three-point (connected) correlation functions factorize as

$$
\left\langle O_{\Delta_{1}}\left(x_{1}\right) O_{\Delta_{2}}\left(x_{2}\right) O_{\Delta_{1}+\Delta_{2}}\left(x_{3}\right)\right\rangle=\mathcal{N}_{\Delta_{1}, \Delta_{2}}\left\langle O_{\Delta_{1}}\left(x_{1}\right) O_{\Delta_{1}}\left(x_{3}\right)\right\rangle\left\langle O_{\Delta_{2}}\left(x_{2}\right) O_{\Delta_{2}}\left(x_{3}\right)\right\rangle ;
$$

where all operators are assumed to be primaries. Consider the $x_{2} \rightarrow x_{1}$ limit. In this limit we can replace $O_{\Delta_{1}}\left(x_{1}\right) O_{\Delta_{2}}\left(x_{2}\right)$ by the OPE, which would express the r.h.s. of (5.9) as a sum over two-point functions.

Let us make the extra assumption that two-point functions of non-identical operators vanish. ${ }^{3}$ The relevant term of the OPE is

$$
O_{\Delta_{1}}\left(x_{1}\right) O_{\Delta_{2}}\left(x_{2}\right)=C_{\Delta_{1} \Delta_{2}} O_{\Delta_{1}+\Delta_{2}}\left(x_{1}\right)+\cdots
$$

Then, substituting into (5.9), one obtains

$$
C_{\Delta_{1} \Delta_{2}}\left\langle O_{\Delta_{1}+\Delta_{2}}\left(x_{1}\right) O_{\Delta_{1}+\Delta_{2}}\left(x_{3}\right)\right\rangle=\mathcal{N}_{\Delta_{1}, \Delta_{2}}\left\langle O_{\Delta_{1}}\left(x_{1}\right) O_{\Delta_{1}}\left(x_{3}\right)\right\rangle\left\langle O_{\Delta_{2}}\left(x_{1}\right) O_{\Delta_{2}}\left(x_{3}\right)\right\rangle .
$$

Equation (5.10) is of the form $F\left(\Delta_{1}+\Delta_{2}\right)=F\left(\Delta_{1}\right) F\left(\Delta_{2}\right)$. This relation "bootstraps" the 2-point function of primary operators of dimension $\Delta$ to be of the form

$$
\left\langle O_{\Delta}(x) O_{\Delta}(0)\right\rangle=\mathcal{C}_{\Delta} e^{-\Delta \ell(x)} .
$$

This is precisely the form of the 2-point function that arises from the geodesic approximation. Thus we see that the factorization property implicitly requires the semiclassical approximation of large $\Delta$. At finite $\Delta$, two-point correlation functions do not have, in general, the form (5.11). An exception is $d=2$, where the exact thermal two-point function is of the form (5.11), in which case the factorization (5.9) also holds for operators of small dimension. For $d>2$, the factorization property for extremal correlators does not generally hold beyond the large $\Delta$ approximation.

On general grounds, $\ell(x)$ must be of the form

$$
\ell(x)=\log |x|^{2}+\sum_{\delta=\delta_{0}} c_{\delta}(T|x|)^{\delta} f_{\delta}(x)
$$

\footnotetext{
${ }^{3}$ At $T \neq 0$ two-point functions of different operators may not vanish. Here we assume that a diagonal basis exists. This seems to be suggested by holography and by the infinite $\beta$ limit.
} 
where $f_{\delta}(x)$ are dimensionless $\mathrm{SO}(d-1)$ invariant functions of $\eta=\frac{\tau}{\sqrt{\tau^{2}+\vec{x}^{2}}}$. Since $\eta \in$ $[-1,1]$, the $f_{\delta}$ can naturally be expressed in terms of Gegenbauer polynomials, which are orthogonal functions in $[-1,1]$. In this way one recovers the general form discussed in [1]. A consequence of the general form (5.11) is that the double scaling limit of [3], $T|x| \rightarrow 0$, $\Delta \rightarrow \infty$, with fixed $\Delta(T|x|)^{\delta_{0}}$, exists and is well defined (in the strong coupling expansion, one has $\delta_{0}=d$ as in (3.12)).

\section{Discussion}

In this paper we have studied 3-point functions in holographic theories at finite temperature concentrating on operators of large conformal dimension. In the gravity dual, they are described by very massive bulk fields. This makes it possible to use the so-called geodesic approximation, which is nothing but the WKB approximation for the bulk equations of motion that determine the bulk-to-boundary propagators. In this approximation, the holographic computation boils down to the study of geodesic arcs in the black-brane background. The geodesic approach has a long history, in particular in trying to identify signatures of the black hole singularity in 2-point functions. However, higher-point functions in finite temperature CFTs have not been thoroughly investigated. One of the motivations of this paper is to fill this gap. We have derived (for the first time) explicit formulas for certain three-point functions in strongly coupled CFT's at finite temperature in $d>2$, and settled the correct equations that describe general three-point functions.

One important technical feature that arises from of our analysis is that, for three (and higher) point functions, one must distinguish two types of geodesics (ingoing/returning), which contribute to the saddle-point equations with different signs, and the correct choice depends on the insertion points of the operators. This observation is new and it is crucial for the correct evaluation of any higher point function $(n>2)$ for large dimension operators.

In general, higher-point correlation functions are computed holographically through Witten diagrams constructed from the interactions in the effective gravity lagrangian. Thus, they critically rely on the particular values and structure of the couplings. However, 3-point functions are special in that only cubic interactions among fluctuations can contribute, and hence they are given by a single Witten diagram, with an overall coupling constant. As a result, the spacetime dependence is given by a universal function of $x_{1}, x_{2}$ (setting $x_{3}=0$ ). While this is obvious at zero temperature - as the 3-point function is fixed by conformal invariance up to a constant -, at $T \neq 0$ it is per se a non-trivial observation in $d>2{ }^{4}$ The further simplification of the geodesic approximation allows to actually evaluate such 3-point functions finding universal predictions for holographic theories. For example, for the symmetric three-point function with $\tau$ dependence we find the universal formula (4.11). For the extremal three-point function, we find

$$
\left\langle O_{\Delta_{1}}\left(x_{1}\right) O_{\Delta_{2}}\left(x_{2}\right) O_{\Delta_{1}+\Delta_{2}}(0)\right\rangle=e^{-\Delta_{1} \ell\left(x_{1}\right)} e^{-\Delta_{2} \ell\left(x_{2}\right)},
$$

\footnotetext{
${ }^{4}$ In $d=2$ one can conformally map $\mathbb{R}^{2}$ to $S^{1} \times \mathbb{R}$. In this case one can argue, solely on field-theoretic grounds, that the 3 -point function must have a fixed structure.
} 
or

$$
\log \left\langle O_{\Delta_{1}}\left(x_{1}\right) O_{\Delta_{2}}\left(x_{2}\right) O_{\Delta_{1}+\Delta_{2}}(0)\right\rangle=-\Delta_{1} \ell\left(x_{1}\right)-\Delta_{2} \ell\left(x_{2}\right),
$$

where the function $\ell(x)$ is universal. It was computed in [3] and it is a complicated function of $T,|x|$ and $\eta=\tau /|x|$. The complete small temperature expansion can be generated in the simplest case when $\vec{x}=0$, where the first few terms are

$$
\ell(\tau)=\frac{\pi^{4} T^{4} \tau^{4}}{40}\left(1+\frac{11}{360} \pi^{4} \tau^{4} T^{4}+\frac{89 \pi^{8} \tau^{8} T^{8}}{46800}+\ldots\right)
$$

For finite $T$, one finds the closed formula $\ell(x)=-\frac{1}{2} \log \left(\mu^{4}+4\right) / 16$, where $\mu=\mu(\tau T)$ is given by a transcendental equation involving logarithms [3].

\subsection{Factorization property as a requirement for holography}

A striking result of our analysis is that extremal 3-point functions for large dimension operators satisfy the factorization property (5.9). It implies an accurate prediction for the behavior of three-point functions in strongly-coupled CFTs at finite temperature that have a holographic dual. Alternatively, the factorization property (5.9) may be viewed as a necessary (and highly non-trivial) condition for a conformal field theory to admit a holographic description in terms of a gravity dual.

The condition applies whenever the CFT has, in its spectrum, a set of three large dimension operators satisfying $\Delta_{3}=\Delta_{1}+\Delta_{2}$ at strong coupling. Consider the simple example of $\lambda \phi^{4}$ theory in $d=4$. The set of operators $\left\{\phi^{n}\right\}$, with positive integer $n$, have classical dimensions $\Delta_{n}=n$, therefore there are operators satisfying $n_{3}=n_{1}+n_{2}$. However, once one-loop anomalous dimensions are taken into account, the condition $\Delta_{3}=\Delta_{1}+\Delta_{2}$ is no longer satisfied. One is interested in this condition at strong $\lambda \gg 1$ coupling. In this limit, anomalous dimensions are not known for this theory and, in principle, there is no reason to expect, just by accident, that there will be operators satisfying $\Delta_{3}=\Delta_{1}+\Delta_{2}$, since $\Delta_{i}$ are continuous (real number) parameters depending on $\lambda$. On the other hand, one can consider a supersymmetric CFT, where $\Delta=$ R-charge is protected by supersymmetry. The relation still holds at finite temperature, since anomalous dimensions depend on the short-distance behavior, which is the same as in the $T=0$ theory. In this case, one can have theories with $\Delta_{3}=\Delta_{1}+\Delta_{2}$ and the factorization property (5.9) provides a condition to test if the CFT can have a holographic dual. Computing a three-point function in stronglycoupled field theory at finite temperature is obviously complicated. However, note that in this case the large dimension approximation is identical to the large charge expansion. This makes it possible, in principle, to apply modern approaches based on large charge expansion that have recently provided new analytic handles into non-perturbative physics (see e.g. [36] for a recent review). It would be very interesting to explore the extension of these techniques to the $T \neq 0$ case. 


\subsection{Bootstrap conditions from factorization}

The general form of the two-point function in finite temperature CFT was derived in [1] and it has the form

$$
\left\langle O_{\Delta}(x) O_{\Delta}(0)\right\rangle=\sum_{\mathcal{O}} a_{B B \mathcal{O}} \frac{|x|^{\Delta_{\mathcal{O}}}}{\beta^{\Delta_{\mathcal{O}}}} C_{J_{\mathcal{O}}}^{(\nu)}(\eta)
$$

Substituting this expansion in (5.10) one can find an infinite set of recursion relations for the OPE coefficients. An example of the type of relations one finds is shown in the appendix B for the case $\Delta_{1}=\Delta_{2}=\Delta$. Assuming a discrete spectrum, one can proceed recursively in increasing dimension. At the "first level" one finds $a_{A A \mathbb{1}}=1$ for all primaries $A$, which is precisely the expected result (to see this, note that $a_{A A \mathbb{1}}$ is the only contributing term if we consider the $\mathbb{R}^{d}$, i.e. $T=0$, correlator). At the second level one finds

$$
a_{B B \mathcal{C}_{1}^{i}}=2 a_{A A \mathcal{C}_{1}^{i}}
$$

This is consistent with

$$
a_{A A \mathcal{C}_{1}^{i}}=\alpha_{i} \Delta_{\mathcal{A}}
$$

which agrees with (3.14) (the first non-trivial term there is $n=1$, and the coefficient is proportional to $\Delta$ ). One can climb up in dimensions, finding new information about OPE coefficients. It becomes increasingly more complicated, as it requires more assumptions about the spectrum. The analysis is interesting, as it goes along the lines of selecting the characteristics that a CFT must have in order to have a gravity dual.

Similarly, one can extract non-trivial information from the much stronger condition (5.9). In particular, it would be very interesting to see what are the constraints imposed on the spectrum, which ultimately may give further insight into the structure of holographic CFT's, including issues such as the discreteness of the spectrum, its spacing or even the identification of the contributing operators.

\section{Acknowledgments}

We thank A. Tseytlin for useful remarks. D.R-G is partially supported by the Spanish government grant MINECO-16-FPA2015-63667-P. He also acknowledges support from the Principado de Asturias through the grant FC-GRUPIN-IDI/2018/000174 J.G.R. acknowledges financial support from projects 2017-SGR-929, MINECO grant PID2019-105614GBC21, and from the State Agency for Research of the Spanish Ministry of Science and Innovation through the "Unit of Excellence María de Maeztu 2020-2023" (CEX2019-000918-M).

\section{A Details on the geodesic arcs}

In this appendix we study each type of geodesic, with particular care to their contribution to the saddle point equations. In this appendix we consider general geodesics with both $\vec{x}$ and $t$ dependence. 
Let us consider a geodesic departing from the boundary of the form $t=t(z), \vec{x}=\vec{x}(z)$. The action is

$$
S=-m \int d z \frac{1}{z} \sqrt{f \dot{t}^{2}-\frac{1}{f}-\dot{\vec{x}}^{2}}
$$

The conjugate momenta to $t$ and $\vec{x}$ (denoted, respectively, by $P_{t} \equiv m \mu$ and $\vec{P} \equiv m \vec{\nu}$ ) are conserved. We choose a coordinate system where $\vec{P}=\left(P_{x}, 0,0\right)$, so the motion is in the subspace $(z, t, x, 0,0)$. It is convenient to introduce the euclidean time $\tau=i t$. Using momentum conservation, we can formally integrate the equations of motion,

$$
\tau=\tau_{1}+I_{\tau}(z), \quad x=x_{1}+I_{x}(z)
$$

where $\tau_{1}$ and $x_{1}$ represent the boundary values and

$$
I_{\tau}(z)=\int_{0}^{z} d z \frac{z \mu}{f \sqrt{f-z^{2} \mu^{2}+f z^{2} \nu^{2}}}, \quad I_{x}(z)=i \int_{0}^{z} d z \frac{z \nu}{\sqrt{f-z^{2} \mu^{2}+f z^{2} \nu^{2}}} .
$$

Note that trajectories that depart from the boundary have imaginary time $t$, therefore real euclidean time $\tau=i t$. As well known, geodesics of physical particles moving in AdS space with real time $t$ and real $\vec{x}$ do not get to the boundary. However, here we are interested in the above "unphysical" geodesics departing from the boundary, which are those that describe the Green's function, as can be shown using the WKB approximation to the Green's equation.

Substituting the solution into the action and using that $m \approx \Delta$, we get

$$
S=-i \Delta \int d z \frac{1}{z \sqrt{f-z^{2} \mu^{2}+f z^{2} \nu^{2}}} .
$$

Geodesics departing from the boundary reach a maximum value $z_{\max }$ and then they return to the boundary, following a "U" shape trajectory. $z_{\max }$ is a solution of

$$
f\left(z_{\max }\right)-z_{\max }^{2} \mu^{2}+f\left(z_{\max }\right) z_{\max }^{2} \nu^{2}=0
$$

and it is the point where $\dot{\tau}$ and $\dot{x}$ are infinity. In computing the three-point function, we shall consider three geodesic arcs meeting at some intersection point $\left(z_{I}, \tau_{I}, x_{I}\right)$. Each arc may meet the intersection point before reaching $z_{\max }$, or in their return way. We will refer to the first type of geodesic as ingoing geodesics, whereas the second type will be called returning geodesics. This distinction is important, because a solution of the saddle-point equations with $0<z_{I}<1$ requires that there must be at least one arc of each type.

For ingoing geodesics, the corresponding action is obtained by integrating from the boundary to the intersecting point

$$
S^{\text {ingoing }}=-i \Delta \int_{\epsilon}^{z_{I}} d z \frac{1}{z \sqrt{f-z^{2} \mu^{2}+f z^{2} \nu^{2}}} .
$$

From the equations for the trajectory, one gets a relation between $z_{I}$ and $\tau_{I}, x_{I}$ :

$$
\tau_{I}^{\text {ingoing }}=\tau_{1}+I_{\tau}^{\text {ingoing }}, \quad x_{I}^{\text {ingoing }}=x_{1}+I_{x}^{\text {ingoing }} ; \quad I_{\tau}^{\text {ingoing }}=I_{\tau}\left(z_{I}\right), \quad I_{x}^{\text {ingoing }}=I_{x}\left(z_{I}\right) .
$$


The action for returning geodesics can be computed by considering the action of a complete geodesic departing the boundary, entering the bulk and coming back to the boundary, subtracting the last piece from $z_{I}$ to the boundary. Thus, we can write

$$
S^{\text {returning }}=2 S^{\max }-S^{\text {ingoing }},
$$

and likewise

$$
\tau=2 \tau^{\max }-\tau^{\text {ingoing }}, \quad x=2 x^{\text {max }}-x^{\text {ingoing }},
$$

where

$$
\begin{aligned}
S^{\max } & =-i \Delta \int_{\epsilon}^{z_{\max }} d z \frac{1}{z \sqrt{f-z^{2} \mu^{2}+f z^{2} \nu^{2}}}, \\
\tau^{\max } & =\tau_{1}+I_{\tau}\left(z_{\max }\right), \quad x^{\max }=x_{1}+I_{x}\left(z_{\max }\right) .
\end{aligned}
$$

The intersection point is obtained by solving the saddle-point equations

$$
\frac{d S}{d z_{I}}=0, \quad \frac{d S}{d \tau_{I}}=0, \quad \frac{d S}{d x_{I}}=0 .
$$

In computing these derivatives, we must take into account the fact that $\mu$ and $\nu$ are functions of $z_{I}, \tau_{I}, x_{I}$ (and of $\tau_{1}, x_{1}$ ) defined by the conditions (A.6).

We will need to use the following identities:

$$
\begin{aligned}
& \frac{\partial S^{\text {ingoing} / \text { returning }}}{\partial \mu}=-i \Delta \mu \frac{\partial I_{\tau}^{\text {ingoing/returning }}}{\partial \mu}+\Delta \nu \frac{\partial I_{x}^{\text {ingoing } / \text { returning }}}{\partial \mu}, \\
& \frac{\partial S^{\text {ingoing } / \text { returning }}}{\partial \nu}=-i \Delta \mu \frac{\partial I_{\tau}^{\text {ingoing/returning }}}{\partial \nu}+\Delta \nu \frac{\partial I_{x}^{\text {ingoing } / \text { returning }}}{\partial \nu} .
\end{aligned}
$$

In addition, by taking the differential of (A.6) (or of the analog equations for the returning case) one obtains the following "constitutive" relations,

$$
\begin{aligned}
\frac{\partial I_{\tau}}{\partial \mu} \frac{\partial \mu}{\partial \tau_{I}}+\frac{\partial I_{\tau}}{\partial \nu} \frac{\partial \nu}{\partial \tau_{I}} & =1, & \frac{\partial I_{x}}{\partial \mu} \frac{\partial \mu}{\partial \tau_{I}}+\frac{\partial I_{x}}{\partial \nu} \frac{\partial \nu}{\partial \tau_{I}} & =0, \\
\frac{\partial I_{\tau}}{\partial \mu} \frac{\partial \mu}{\partial x_{I}}+\frac{\partial I_{\tau}}{\partial \nu} \frac{\partial \nu}{\partial x_{I}} & =0, & \frac{\partial I_{x}}{\partial \mu} \frac{\partial \mu}{\partial x_{I}}+\frac{\partial I_{x}}{\partial \nu} \frac{\partial \nu}{\partial x_{I}} & =1, \\
\frac{\partial I_{\tau}}{\partial z_{I}}+\frac{\partial I_{\tau}}{\partial \mu} \frac{\partial \mu}{\partial z_{I}}+\frac{\partial I_{\tau}}{\partial \nu} \frac{\partial \nu}{\partial z_{I}} & =0, & \frac{\partial I_{x}}{\partial z_{I}}+\frac{\partial I_{x}}{\partial \mu} \frac{\partial \mu}{\partial z_{I}}+\frac{\partial I_{x}}{\partial \nu} \frac{\partial \nu}{\partial z_{I}} & =0 .
\end{aligned}
$$

\section{A.1 The $z_{I}$ saddle-point equation}

The contribution to the saddle-point equation in $z_{I}$ is

$$
\frac{d S}{d z_{I}}=\frac{\partial S}{\partial z_{I}}+\frac{\partial S}{\partial \mu} \frac{\partial \mu}{\partial z_{I}}+\frac{\partial S}{\partial \nu} \frac{\partial \nu}{\partial z_{I}}
$$

Using (A.16), one gets

$$
\frac{d S}{d z_{I}}=\frac{\partial S}{\partial z_{I}}+\frac{\frac{\partial S}{\partial \mu} \frac{\partial I_{x}}{\partial \nu}-\frac{\partial S}{\partial \nu} \frac{\partial I_{x}}{\partial \mu}}{\frac{\partial I_{x}}{\partial \mu} \frac{\partial I_{\tau}}{\partial \nu}-\frac{\partial I_{\tau}}{\partial \mu} \frac{\partial I_{x}}{\partial \nu}} \frac{\partial I_{\tau}}{\partial z_{I}}+\frac{\frac{\partial S}{\partial \nu} \frac{\partial I_{\tau}}{\partial \mu}-\frac{\partial S}{\partial \mu} \frac{\partial I_{\tau}}{\partial \nu}}{\frac{\partial I_{x}}{\partial \mu} \frac{\partial I_{\tau}}{\partial \nu}-\frac{\partial I_{\tau}}{\partial \mu} \frac{\partial I_{x}}{\partial \nu}} \frac{\partial I_{x}}{\partial z_{I}} .
$$


Using now (A.12), (A.13), this becomes

$$
\frac{d S}{d z_{I}}=\frac{\partial S}{\partial z_{I}}+i \Delta \mu \frac{\partial I_{\tau}}{\partial z_{I}}-\Delta \nu \frac{\partial I_{x}}{\partial z_{I}} .
$$

Using now the explicit expressions of $S, I_{\tau}$ and $I_{x}$, we finally get

$$
\frac{d S}{d z_{I}}=-\frac{i \epsilon \Delta}{z_{I} f\left(z_{I}\right)} \sqrt{f\left(z_{I}\right)-z_{I}^{2} \mu^{2}+f\left(z_{I}\right) z_{I}^{2} \nu^{2}},
$$

where $\epsilon=1$ for ingoing geodesics and $\epsilon=-1$ for returning geodesics. The complete saddlepoint equation for $z_{I}$ is obtained by adding the contributions (A.20) from the different geodesic arcs.

\section{A.2 The saddle-point equations for $x_{I}$ and $\tau_{I}$}

Since $S$ has no explicit $x_{I}, \tau_{I}$ dependence, we have

$$
\frac{d S}{d X}=\frac{\partial S}{\partial \mu} \frac{\partial \mu}{\partial X}+\frac{\partial S}{\partial \nu} \frac{\partial \nu}{\partial X},
$$

where $X$ is either $x_{I}$ or $\tau_{I}$. Using (A.14) and (A.15), one finds

$$
\frac{d S}{d \tau_{I}}=\frac{\frac{\partial I_{x}}{\partial \mu} \frac{\partial S}{\partial \nu}-\frac{\partial I_{x}}{\partial \nu} \frac{\partial S}{\partial \mu}}{\frac{\partial I_{x}}{\partial \mu} \frac{\partial I_{\tau}}{\partial \nu}-\frac{\partial I_{\tau}}{\partial \mu} \frac{\partial I_{x}}{\partial \nu}}, \quad \frac{d S}{d x_{I}}=\frac{\frac{\partial I_{\tau}}{\partial \nu} \frac{\partial S}{\partial \mu}-\frac{\partial I_{\tau}}{\partial \mu} \frac{\partial S}{\partial \nu}}{\frac{\partial I_{x}}{\partial \mu} \frac{\partial I_{\tau}}{\partial \nu}-\frac{\partial I_{\tau}}{\partial \mu} \frac{\partial I_{x}}{\partial \nu}} .
$$

Finally, using the identities in (A.12), (A.13), we obtain

$$
\frac{d S}{d \tau_{I}}=-i \Delta \mu, \quad \frac{d S}{d x_{I}}=\Delta \nu .
$$

By adding the contributions (A.23) for the different geodesic arcs, we obtain the general saddle-point equations (5.6) anticipated in section 5.2.

\section{B Conditions on OPE coefficients from factorization}

In this appendix we initiate the exploration of the conditions that the factorization property imposes on 2-point functions. As discussed in the main text, the factorization equation (5.9) implies (5.10) (under the assumption, valid within the limits of the holographic approximation, that off-diagonal correlators, i.e. those of operators of different dimensions, vanish). Although (5.10) contains less information than (5.9), as it is a particular case where $x_{2} \rightarrow x_{1}$, here we will examine some consequences of (5.10), which provides a much simpler starting point.

Consider for instance the case $\Delta_{1}=\Delta_{2}=\Delta, \Delta_{3}=2 \Delta$ and choose $x_{3}=0$. Then (5.10) becomes $\left(C_{\Delta}\right.$ collects all the constants)

$$
\left\langle O_{2 \Delta}(x) O_{2 \Delta}(0)\right\rangle=C_{\Delta}\left\langle O_{\Delta}(x) O_{\Delta}(0)\right\rangle^{2} .
$$

This gives the relation

$$
\sum_{\mathcal{O}} a_{B B \mathcal{O}} \frac{|x|^{\Delta_{\mathcal{O}}}}{\beta^{\Delta_{\mathcal{O}}}} C_{J_{\mathcal{O}}}^{(\nu)}(\eta)=\sum_{\mathcal{A}, \mathcal{B}} a_{A A \mathcal{A}} a_{A A \mathcal{B}} \frac{|x|^{\Delta_{\mathcal{A}}+\Delta_{\mathcal{B}}}}{\beta^{\Delta_{\mathcal{A}}+\Delta_{\mathcal{B}}}} C_{J_{\mathcal{A}}}^{(\nu)} C_{J_{\mathcal{B}}}^{(\nu)}
$$


where $A=O_{\Delta}$ and $B=O_{2 \Delta}$. The Gegenbauer polynomials are orthogonal functions in $[-1,1]$. They satisfy

$$
\int_{-1}^{1} d \eta\left(1-\eta^{2}\right)^{\nu-\frac{1}{2}} C_{J_{1}}^{(\nu)}(\eta) C_{J_{2}}^{(\nu)}(\eta)=\mathcal{N}_{J_{1}} \delta_{J_{1}, J_{2}}
$$

and

$$
\int_{-1}^{1} d \eta\left(1-\eta^{2}\right)^{\nu-\frac{1}{2}} C_{J_{1}}^{(\nu)}(\eta) C_{J_{2}}^{(\nu)}(\eta) C_{J_{3}}^{(\nu)}(\eta)=f_{J_{1}, J_{2}, J_{3}}
$$

where $\mathcal{N}_{J_{1}}$ and $f_{J_{1}, J_{2}, J_{3}}$ are known factors. For our purposes now the relevant fact is that $f_{J_{1}, J_{2}, J_{3}}$ is not vanishing only if

$$
f_{J_{1}, J_{2}, J_{3}} \neq 0 \text { if }\left\{\begin{array}{l}
J_{1} \leq J_{2}+J_{3} \& J_{2} \leq J_{1}+J_{3} \& J_{3} \leq J_{1}+J_{2} \\
J_{1}+J_{2}+J_{3}=\text { even }
\end{array}\right.
$$

Using this property, we find that the bootstrap equation requires that

$$
\mathcal{N}_{J_{\mathcal{C}}} a_{B B \mathcal{C}}=\sum_{\mathcal{A}, \mathcal{B}} a_{A A \mathcal{A}} a_{A A \mathcal{B}} \frac{|x|^{\Delta_{\mathcal{A}}+\Delta_{\mathcal{B}}-\Delta_{\mathcal{C}}}}{\beta^{\Delta_{\mathcal{A}}+\Delta_{\mathcal{B}}-\Delta_{\mathcal{C}}}} f_{J_{\mathcal{A}}, J_{\mathcal{B}}, J_{\mathcal{C}}}
$$

Let us define $\varrho=\frac{|x|}{\beta}$ and separate the $\varrho$-independent term

$$
\begin{aligned}
\mathcal{N}_{J_{\mathcal{C}}} a_{B B \mathcal{C}}= & \sum_{\mathcal{A}, \mathcal{B}} a_{A A \mathcal{A}} a_{A A \mathcal{B}} f_{J_{\mathcal{A}}, J_{\mathcal{B}}, J_{\mathcal{C}}} \delta_{\Delta_{\mathcal{A}}+\Delta_{\mathcal{B}}, \Delta_{\mathcal{C}}} \\
& +\sum_{\mathcal{A}, \mathcal{B}} a_{A A \mathcal{A}} a_{A A \mathcal{B}} \varrho^{\Delta_{\mathcal{A}}+\Delta_{\mathcal{B}}-\Delta_{\mathcal{C}}} f_{J_{\mathcal{A}}, J_{\mathcal{B}}, J_{\mathcal{C}}}\left(1-\delta_{\Delta_{\mathcal{A}}+\Delta_{\mathcal{B}}, \Delta_{\mathcal{C}}}\right)
\end{aligned}
$$

The l.h.s. does not depend on $\varrho$, therefore it must equal the first term,

$$
\mathcal{N}_{J_{\mathcal{C}}} a_{B B \mathcal{C}}=\sum_{\mathcal{A}, \mathcal{B}} a_{A A \mathcal{A}} a_{A A \mathcal{B}} f_{J_{\mathcal{A}}, J_{\mathcal{B}}, J_{\mathcal{C}}} \delta_{\Delta_{\mathcal{A}}+\Delta_{\mathcal{B}, \Delta_{\mathcal{C}}}}
$$

Let us consider the operator $\mathcal{C}=\mathbb{1}$. In that case $f_{J_{1}, J_{2}, 0}=\mathcal{N}_{J_{1}} \delta_{J_{1}, J_{2}}$. Then

$$
\mathcal{N}_{0} a_{B B \mathbb{1}}=\sum_{\mathcal{A}, \mathcal{B}} \mathcal{N}_{J_{\mathcal{A}}} a_{A A \mathcal{A}} a_{A A \mathcal{B}} \delta_{J_{\mathcal{A}}, J_{\mathcal{B}}} \delta_{\Delta_{\mathcal{A}}+\Delta_{\mathcal{B}}, 0}
$$

Obviously this is only satisfied for $\mathcal{A}=\mathcal{B}=\mathbb{1}$, when this equation becomes

$$
a_{B B \mathbb{1}}=a_{A A \mathbb{1}}^{2}
$$

whose solution is $a_{A A \mathbb{1}}=1$ for all primaries $A$, as expected.

Going beyond requires making some assumptions on the spectrum of the theory. To begin with, let us assume that the CFT has a gapped spectrum, and consider the next "level" beyond the identity operator. Let us call the operators there $\mathcal{C}_{1}^{i}-i$ is an unspecified index, as there may be more than one. Then

$$
\mathcal{N}_{J_{\mathcal{C}_{1}^{i}}} a_{B B \mathcal{C}_{1}^{i}}=\sum_{\mathcal{A}, \mathcal{B}} a_{A A \mathcal{A}} a_{A A \mathcal{B}} f_{J_{\mathcal{A}}, J_{\mathcal{B}}, J_{\mathcal{C}_{1}^{i}}} \delta_{\Delta_{\mathcal{A}}+\Delta_{\mathcal{B}}, \Delta_{\mathcal{C}_{1}^{i}}}
$$


It is clear that it can only be that either $\mathcal{A}=\mathcal{C}_{1}^{j}$ and $\mathcal{B}=\mathbb{1}$ or vice-versa. Thus, this gives

$$
\mathcal{N}_{J_{\mathcal{C}_{1}^{i}}} a_{B B \mathcal{C}_{1}^{i}}=2 \sum_{j} a_{A A \mathcal{C}_{1}^{j}} f_{J_{\mathcal{C}_{1}^{j}}, J_{\mathcal{C}_{1}^{i}}}
$$

where we have used that $a_{A A \mathbb{1}}=1$. Let us assume that, with a given spin and dimension, there is one single operator. Then, the above equation becomes

$$
a_{B B \mathcal{C}_{1}^{i}}=2 a_{A A \mathcal{C}_{1}^{i}}
$$

This is consistent with $a_{A A C_{1}^{i}}=\alpha_{i} \Delta_{\mathcal{A}}$ for a generic $A$, which, as described in the main text, would be compatible with our results.

One could carry on this program to higher "levels". However it becomes increasingly complicated, as in particular one has to make more and more hypothesis on the spectrum of the theory.

Open Access. This article is distributed under the terms of the Creative Commons Attribution License (CC-BY 4.0), which permits any use, distribution and reproduction in any medium, provided the original author(s) and source are credited.

\section{References}

[1] L. Iliesiu, M. Koloğlu, R. Mahajan, E. Perlmutter and D. Simmons-Duffin, The Conformal Bootstrap at Finite Temperature, JHEP 10 (2018) 070 [arXiv:1802.10266] [INSPIRE].

[2] S. El-Showk and K. Papadodimas, Emergent Spacetime and Holographic CFTs, JHEP 10 (2012) 106 [arXiv:1101.4163] [InSPIRE].

[3] D. Rodriguez-Gomez and J.G. Russo, Correlation functions in finite temperature CFT and black hole singularities, JHEP 06 (2021) 048 [arXiv: 2102.11891] [INSPIRE].

[4] L.F. Alday, M. Kologlu and A. Zhiboedov, Holographic correlators at finite temperature, JHEP 06 (2021) 082 [arXiv:2009.10062] [INSPIRE].

[5] N. Lashkari, A. Dymarsky and H. Liu, Eigenstate Thermalization Hypothesis in Conformal Field Theory, J. Stat. Mech. 1803 (2018) 033101 [arXiv:1610.00302] [INSPIRE].

[6] A.L. Fitzpatrick and K.-W. Huang, Universal Lowest-Twist in CFTs from Holography, JHEP 08 (2019) 138 [arXiv: 1903.05306] [INSPIRE].

[7] A.L. Fitzpatrick, K.-W. Huang and D. Li, Probing universalities in d > 2 CFTs: from black holes to shockwaves, JHEP 11 (2019) 139 [arXiv:1907.10810] [INSPIRE].

[8] R. Karlsson, A. Parnachev and P. Tadić, Thermalization in Large-N CFTs, arXiv:2102.04953 [INSPIRE].

[9] T. Klose and T. McLoughlin, A light-cone approach to three-point functions in $A d S_{5} \times S^{5}$, JHEP 04 (2012) 080 [arXiv:1106.0495] [INSPIRE].

[10] E.I. Buchbinder and A.A. Tseytlin, Semiclassical correlators of three states with large $S^{5}$ charges in string theory in $A d S_{5} \times S^{5}$, Phys. Rev. D 85 (2012) 026001 [arXiv:1110.5621] [INSPIRE].

[11] J.A. Minahan, Holographic three-point functions for short operators, JHEP 07 (2012) 187 [arXiv: 1206.3129] [INSPIRE]. 
[12] A. Dymarsky and M. Smolkin, Krylov complexity in conformal field theory, Phys. Rev. D 104 (2021) L081702 [arXiv: 2104 .09514] [INSPIRE].

[13] P. Kraus, H. Ooguri and S. Shenker, Inside the horizon with AdS/CFT, Phys. Rev. D 67 (2003) 124022 [hep-th/0212277] [INSPIRE].

[14] L. Fidkowski, V. Hubeny, M. Kleban and S. Shenker, The Black hole singularity in AdS/CFT, JHEP 02 (2004) 014 [hep-th/0306170] [INSPIRE].

[15] A. Hamilton, D.N. Kabat, G. Lifschytz and D.A. Lowe, Local bulk operators in AdS/CFT: A Holographic description of the black hole interior, Phys. Rev. D 75 (2007) 106001 [Erratum ibid. 75 (2007) 129902] [hep-th/0612053] [INSPIRE].

[16] V. Balasubramanian et al., Holographic Thermalization, Phys. Rev. D 84 (2011) 026010 [arXiv: 1103.2683] [INSPIRE].

[17] I. Heemskerk, D. Marolf, J. Polchinski and J. Sully, Bulk and Transhorizon Measurements in AdS/CFT, JHEP 10 (2012) 165 [arXiv:1201.3664] [INSPIRE].

[18] K. Papadodimas and S. Raju, An Infalling Observer in AdS/CFT, JHEP 10 (2013) 212 [arXiv: 1211.6767] [INSPIRE].

[19] T. Hartman and J. Maldacena, Time Evolution of Entanglement Entropy from Black Hole Interiors, JHEP 05 (2013) 014 [arXiv:1303.1080] [INSPIRE].

[20] M. Dodelson and H. Ooguri, Singularities of thermal correlators at strong coupling, Phys. Rev. D 103 (2021) 066018 [arXiv:2010.09734] [inSPIRE].

[21] M. Grinberg and J. Maldacena, Proper time to the black hole singularity from thermal one-point functions, JHEP 03 (2021) 131 [arXiv:2011.01004] [INSPIRE].

[22] S.S. Gubser, I.R. Klebanov and A.A. Tseytlin, Coupling constant dependence in the thermodynamics of $N=4$ supersymmetric Yang-Mills theory, Nucl. Phys. B 534 (1998) 202 [hep-th/9805156] [INSPIRE].

[23] H. Osborn and A.C. Petkou, Implications of conformal invariance in field theories for general dimensions, Annals Phys. 231 (1994) 311 [hep-th/9307010] [INSPIRE].

[24] F. Bastianelli, S. Frolov and A.A. Tseytlin, Three point correlators of stress tensors in maximally supersymmetric conformal theories in $D=3$ and $D=6$, Nucl. Phys. B $\mathbf{5 7 8}$ (2000) 139 [hep-th/9911135] [INSPIRE].

[25] S.M. Chester, J. Lee, S.S. Pufu and R. Yacoby, The $\mathcal{N}=8$ superconformal bootstrap in three dimensions, JHEP 09 (2014) 143 [arXiv: 1406.4814] [INSPIRE].

[26] S. Dobashi, H. Shimada and T. Yoneya, Holographic reformulation of string theory on $A d S_{5} \times S^{5}$ background in the PP wave limit, Nucl. Phys. B 665 (2003) 94 [hep-th/0209251] [INSPIRE].

[27] S. Dobashi and T. Yoneya, Resolving the holography in the plane-wave limit of AdS/CFT correspondence, Nucl. Phys. B 711 (2005) 3 [hep-th/0406225] [INSPIRE].

[28] R.A. Janik, P. Surowka and A. Wereszczynski, On correlation functions of operators dual to classical spinning string states, JHEP 05 (2010) 030 [arXiv: 1002.4613] [INSPIRE].

[29] Y. Gobeil, A. Maloney, G.S. Ng and J.-q. Wu, Thermal Conformal Blocks, SciPost Phys. 7 (2019) 015 [arXiv : 1802.10537] [inSPIRE].

[30] P. Kovtun and A. Ritz, Black holes and universality classes of critical points, Phys. Rev. Lett. 100 (2008) 171606 [arXiv:0801.2785] [INSPIRE]. 
[31] I.R. Klebanov and A.A. Tseytlin, Entropy of near extremal black p-branes, Nucl. Phys. B 475 (1996) 164 [hep-th/9604089] [INSPIRE].

[32] M. Becker, Y. Cabrera and N. Su, Finite-temperature three-point function in $2 D$ CFT, JHEP 09 (2014) 157 [arXiv: 1407.3415] [inSPIRE].

[33] S. Lee, S. Minwalla, M. Rangamani and N. Seiberg, Three point functions of chiral operators in $D=4, N=4 S Y M$ at large $N$, Adv. Theor. Math. Phys. 2 (1998) 697 [hep-th/9806074] [INSPIRE].

[34] H. Liu and A.A. Tseytlin, Dilaton-fixed scalar correlators and $A d S_{5} \times S^{5}-S Y M$ correspondence, JHEP 10 (1999) 003 [hep-th/9906151] [INSPIRE].

[35] E. D'Hoker, D.Z. Freedman, S.D. Mathur, A. Matusis and L. Rastelli, Extremal correlators in the AdS/CFT correspondence, hep-th/9908160 [INSPIRE].

[36] L.A. Gaumé, D. Orlando and S. Reffert, Selected topics in the large quantum number expansion, Phys. Rept. 933 (2021) 2180 [arXiv: 2008.03308] [INSPIRE]. 